TRANSACTIONS OF THE

AMERICAN MATHEMATICAL SOCIETY

Volume 362, Number 10, October 2010, Pages 5455-5481

S 0002-9947(10)05166-4

Article electronically published on May 3, 2010

\title{
AN EXCELLENT F-PURE RING OF PRIME CHARACTERISTIC HAS A BIG TIGHT CLOSURE TEST ELEMENT
}

\author{
RODNEY Y. SHARP
}

\begin{abstract}
In two recent papers, the author has developed a theory of graded annihilators of left modules over the Frobenius skew polynomial ring over a commutative Noetherian ring $R$ of prime characteristic $p$, and has shown that this theory is relevant to the theory of test elements in tight closure theory. One result of that work was that, if $R$ is local and the $R$-module structure on the injective envelope $E$ of the simple $R$-module can be extended to a structure as a torsion-free left module over the Frobenius skew polynomial ring, then $R$ is $F$-pure and has a tight closure test element. One of the central results of this paper is the converse, namely that, if $R$ is $F$-pure, then $E$ has a structure as a torsion-free left module over the Frobenius skew polynomial ring; a corollary is that every $F$-pure local ring of prime characteristic, even if it is not excellent, has a tight closure test element. These results are then used, along with embedding theorems for modules over the Frobenius skew polynomial ring, to show that every excellent (not necessarily local) $F$-pure ring of characteristic $p$ must have a so-called 'big' test element.
\end{abstract}

\section{INTRODUCTION}

This paper is concerned with the existence of tight closure test elements for an $F$-pure (commutative Noetherian) ring of prime characteristic.

In two recent papers [15] and [16], the author has developed a theory of graded annihilators of left modules over the Frobenius skew polynomial ring (over a commutative Noetherian ring of prime characteristic), and has shown that this theory is relevant to the theory of tight closure test elements. We shall therefore recall, in this Introduction, certain definitions from M. Hochster's and C. Huneke's theory of tight closure, but they will be presented below in terms of the Frobenius skew polynomial ring.

Throughout the paper, $R$ will denote a commutative Noetherian ring of prime characteristic $p$. We shall only assume that $R$ is local when this is explicitly stated; then, the notation ' $(R, \mathfrak{m})$ ' will denote that $\mathfrak{m}$ is the maximal ideal of $R$. We shall always denote by $f: R \longrightarrow R$ the Frobenius homomorphism, for which $f(r)=r^{p}$ for all $r \in R$. The Frobenius skew polynomial ring over $R$ is the skew polynomial ring $R[x, f]$ associated to $R$ and $f$ in the indeterminate $x$ over $R$. Recall that $R[x, f]$ is, as a left $R$-module, freely generated by $\left(x^{i}\right)_{i \in \mathbb{N}_{0}}$ (throughout the paper, we use $\mathbb{N}$ and $\mathbb{N}_{0}$ to denote the set of positive integers and the set of non-negative integers,

Received by the editors December 18, 2008.

2010 Mathematics Subject Classification. Primary 13A35, 16S36, 13D45, 13E05, 13E10, 13H10; Secondary 13J10.

Key words and phrases. Commutative Noetherian ring, prime characteristic, Frobenius homomorphism, tight closure, test element, $F$-pure ring, Frobenius skew polynomial ring. 
respectively), and so consists of all polynomials $\sum_{i=0}^{n} r_{i} x^{i}$, where $n \in \mathbb{N}_{0}$ and $r_{0}, \ldots, r_{n} \in R$; however, its multiplication is subject to the rule $x r=f(r) x=r^{p} x$ for all $r \in R$. Note that $R[x, f]$ can be considered as a positively-graded ring $R[x, f]=\bigoplus_{n=0}^{\infty} R[x, f]_{n}$, with $R[x, f]_{n}=R x^{n}$ for all $n \in \mathbb{N}_{0}$.

If, for $n \in \mathbb{N}$, we endow $R x^{n}$ with its natural structure as an $(R, R)$-bimodule (inherited from its being a graded component of $R[x, f]$ ), then $R x^{n}$ is isomorphic (as an $(R, R)$-bimodule) to $R$ viewed as a left $R$-module in the natural way and as a right $R$-module via $f^{n}$, the $n$th iterate of the Frobenius ring homomorphism. We can now write that $R$ is $F$-pure precisely when, for each $R$-module $N$, the map $\psi_{N}: N \longrightarrow R x \otimes_{R} N$ for which $\psi_{N}(g)=x \otimes g$ for all $g \in N$ is injective.

A left $R[x, f]$-module $H$ is said to be $x$-torsion-free precisely when

$$
\Gamma_{x}(H):=\left\{h \in H: x^{n} h=0 \text { for some } n \in \mathbb{N}\right\}=0 .
$$

It is worth pointing out right away that, when $R$ is $F$-pure, the left $R[x, f]$-module $R[x, f] \otimes_{R} N$ is $x$-torsion-free, for each $R$-module $N$.

We now formulate some of the basic definitions of tight closure theory in terms of the Frobenius skew polynomial ring. The theory of tight closure was invented by M. Hochster and C. Huneke [5], and many applications have been found for the theory; see 8 .

We use $R^{\circ}$ to denote the complement in $R$ of the union of the minimal prime ideals of $R$. Let $L$ and $M$ be $R$-modules and let $K$ be a submodule of $L$. Observe that there is a natural structure as an $\mathbb{N}_{0}$-graded left $R[x, f]$-module on

$$
R[x, f] \otimes_{R} M=\bigoplus_{n \in \mathbb{N}_{0}}\left(R x^{n} \otimes_{R} M\right) .
$$

An element $m \in M$ belongs to $0_{M}^{*}$, the tight closure of the zero submodule in $M$, if and only if there exists $c \in R^{\circ}$ such that the element $1 \otimes m \in\left(R[x, f] \otimes_{R} M\right)_{0}$ is annihilated by $c x^{j}$ for all $j \gg 0$; see Hochster-Huneke [5, §8]. (Incidentally, in discussions of this type, we shall often tacitly identify

$$
\left(R[x, f] \otimes_{R} M\right)_{0}=R x^{0} \otimes_{R} M=R \otimes_{R} M
$$

with $M$ in the obvious way.) Furthermore, the tight closure $K_{L}^{*}$ of $K$ in $L$ is the inverse image, under the natural epimorphism $L \longrightarrow L / K$, of $0_{L / K}^{*}$, the tight closure of 0 in $L / K$. In general, we have $K \subseteq K_{L}^{*}$; we say that $K$ is tightly closed in $L$ precisely when $K=K_{L}^{*}$, that is, if and only if $0_{L / K}^{*}=0$.

The ring $R$ is said to be weakly $F$-regular precisely when every ideal of $R$ is tightly closed in $R$, and to be $F$-regular if and only if each ring of fractions $S^{-1} R$ of $R$ with respect to an arbitrary multiplicatively closed subset $S$ of $R$ is weakly $F$-regular.

A test element for modules for $R$ is an element $c \in R^{\circ}$ such that, for every finitely generated $R$-module $M$ and every $j \in \mathbb{N}_{0}$, the element $c x^{j}$ annihilates $1 \otimes m \in\left(R[x, f] \otimes_{R} M\right)_{0}$ for every $m \in 0_{M}^{*}$. The phrase 'for modules' is inserted because Hochster and Huneke have also considered a concept of a test element for ideals for $R$, which is defined to be an element $c \in R^{\circ}$ such that, for every cyclic $R$-module $M$ and every $j \in \mathbb{N}_{0}$, the element $c x^{j}$ annihilates $1 \otimes m \in\left(R[x, f] \otimes_{R} M\right)_{0}$ for every $m \in 0_{M}^{*}$. When $R$ is reduced and excellent, the concepts of a test element for modules and a test element for ideals for $R$ coincide; see 5. Discussion (8.6) and Proposition (8.15)]. With this, and the fact that the main existence results for test elements in this paper only apply to excellent rings that are $F$-pure (and therefore 
reduced), in mind, we shall use the phrase 'test element for $R$ ' as an abbreviation for 'test element for modules for $R$ '.

A big test element for $R$ is defined to be an element $c \in R^{\circ}$ such that, for every $R$-module $M$ and every $j \in \mathbb{N}_{0}$, the element $c x^{j}$ annihilates $1 \otimes m \in\left(R[x, f] \otimes_{R} M\right)_{0}$ for every $m \in 0_{M}^{*}$.

It is generally accepted that, currently, the best-known results about the existence of test elements are those of Hochster-Huneke in [6, §6]. Here is one of their results. (For $c \in R$, we denote by $R_{c}$ the ring of fractions of $R$ with respect to the multiplicatively closed set consisting of the powers of $c$.)

0.1. Theorem (M. Hochster and C. Huneke [6, Theorem (6.1)(b)]). A reduced algebra $R$ of finite type over an excellent local ring of characteristic $p$ has a test element.

In fact, if $c \in R^{\circ}$ is such that $R_{c}$ is regular, then some power of $c$ is a test element for $R$.

However, it is also generally accepted that the proof of the above theorem in [6] is quite difficult, because it depends on the so-called ' $\Gamma$-construction'.

One of the main results of [16] is that, if $(R, \mathfrak{m})$ is local and the $R$-module structure on the injective envelope $E_{R}(R / \mathfrak{m})$ of $R / \mathfrak{m}$ can be extended to an $x$ torsion-free left $R[x, f]$-module structure, then $R$ is $F$-pure and has a test element for modules. In this paper we shall prove the converse, and so obtain (in Theorem 3.2 below) the following characterization of $F$-pure local rings.

Theorem. Suppose that $(R, \mathfrak{m})$ is local. Then $R$ is $F$-pure if and only if the $R$ module structure on $E_{R}(R / \mathfrak{m})$ can be extended to an $x$-torsion-free left $R[x, f]$ module structure.

A corollary is that every $F$-pure local ring (such a ring must be reduced) has a test element for modules, even if it is not excellent.

The methods from [15] and [16] based on graded annihilators are particularly well-suited to $x$-torsion-free left $R[x, f]$-modules. A key point is that those methods associate, with an $x$-torsion-free left $R[x, f]$-module $E$ that is Artinian as an $R$ module, a certain finite set $\mathcal{I}(E)$ of radical ideals of $R$, and, in some circumstances (such as the case where $(R, \mathfrak{m})$ is local and $E$ is $R$-isomorphic to the injective envelope of the simple $R$-module), this set $\mathcal{I}(E)$ has connections with tight closure test elements.

This paper uses those ideas, in conjunction with embedding theorems for modules over the Frobenius skew polynomial ring, to prove (in Theorem 4.16 below) the following existence theorem.

Theorem. Suppose that $R$ is F-pure and excellent (but not necessarily local). Then $R$ has a big test element.

In fact, any $c \in R^{\circ}$ for which $R_{c}$ is regular must be a big test element for $R$.

This theorem only applies to $F$-pure excellent rings, and so has rather limited applicability compared with the Hochster-Huneke Theorem 0.1 on the other hand, it does apply to all $F$-pure excellent rings and not just to those $F$-pure rings that are algebras of finite type over an excellent local ring. Also, the theorem asserts the existence of big test elements, as opposed to test elements. In fact, one can deduce fairly quickly from known results of Hochster and Huneke that, if $R$ is $F$-pure and excellent and $c \in R^{\circ}$ is such that $R_{c}$ is regular, then $c$ is a test element for $R$. 
We prove this claim now. However, the reader should note that the main aim of this paper is to show that methods based on graded annihilators from [15] and [16] provide a completely new approach to questions about the existence of tight closure test elements.

0.2. Theorem. Suppose that $R$ is F-pure and excellent (but not necessarily local). Then $R$ has a test element.

In fact, any $c \in R^{\circ}$ for which $R_{c}$ is regular must be a test element for $R$.

Proof. Since $F$-pure rings are reduced, the concepts of 'test element for modules' and 'test element for ideals' coincide for $R$; see Hochster-Huneke [5, Discussion (8.6) and Proposition (8.15)]. By Hochster-Huneke [5, Proposition 6.1(a)], it is enough, in order to show that $c$ is a test element for $R$, to show that $c / 1 \in R_{\mathfrak{p}}$ is a test element for $R_{\mathfrak{p}}$ for each prime ideal $\mathfrak{p}$ of $R$.

By Hochster and J. L. Roberts [7, Lemma 6.2], the localization $R_{\mathfrak{p}}$ is again $F$ pure; of course, $R_{\mathfrak{p}}$ is excellent. Furthermore, since $\left(R_{\mathfrak{p}}\right)_{c / 1}$, the ring of fractions of $R_{\mathfrak{p}}$ with respect to the set of powers of the element $c / 1$ of $\left(R_{\mathfrak{p}}\right)^{\circ}$, is a ring of fractions of $R_{c}$, it is regular. It is therefore enough for us to prove the claim in the case where $(R, \mathfrak{m})$ is local.

In that case, we can appeal to the Hochster-Huneke Theorem 0.1 to deduce the existence of $e \in \mathbb{N}_{0}$ such that $c^{p^{e}}$ is a test element for $R$. We shall now deduce that $c$ itself is a test element for $R$.

Let $M$ be a finitely generated $R$-module, and let $m \in 0_{M}^{*}$. Therefore

$$
c^{p^{e}} x^{j}(1 \otimes m)=0
$$

in $R[x, f] \otimes_{R} M$ for all $j \in \mathbb{N}_{0}$. Thus

$$
x^{e} c x^{i}(1 \otimes m)=c^{p^{e}} x^{e+i}(1 \otimes m)=0 \quad \text { for all } i \in \mathbb{N}_{0} .
$$

However, $R[x, f] \otimes_{R} M$ is $x$-torsion-free because $R$ is $F$-pure, and so $c x^{i}(1 \otimes m)=0$ for all $i \in \mathbb{N}_{0}$. This is true for all $m \in 0_{M}^{*}$, for each finitely generated $R$-module $M$. Therefore $c$ is a test element for $R$.

The main aim of this paper is to show that the theory of graded annihilators can be used to strengthen the first paragraph of Theorem 0.2 by the insertion of 'big' just before 'test element', and thereby provide an alternative proof that is independent of the Hochster-Huneke Theorem 0.1 and the $\Gamma$-construction.

It is a pleasure to record my gratitude to Mordechai Katzman for many discussions about matters related to the work in this paper, and particularly about the material in $\S 2$ below.

Note. The referee has asked the author to report that results of Hochster and Huneke on test elements have been extended by H. Elitzur in his unpublished 2003 University of Michigan thesis 'Tight closure in Artinian modules'. As Elitzur's work in this context has not been published, the author will rely completely on the referee for the accuracy of the comments in this note.

Elitzur defines a 'general test element for $R$ ' to be an element $c \in R^{\circ}$ such that, for every $R$-module $L$ and every submodule $K$ of $L$, an element $m$ of $L$ belongs to $K_{L}^{*}$ if and only if $c x^{j}$ annihilates $1 \otimes(m+K) \in\left(R[x, f] \otimes_{R}(L / K)\right)_{0}$ for all $j \gg 0$. The referee reports that Elitzur's results about general test elements are stated for F-finite rings, and that Proposition 3.4 of Elitzur's thesis uses Hochster's and Huneke's [6, Theorem 5.10] to produce general test elements. 


\section{Some NOTATION AND KNOWN RESUlts}

This paper builds on the results of [15] and [16], and we shall make much use of notation, terminology and results from [15, §1] and [16, §1].

1.1. Notation. Let $H$ be a left $R[x, f]$-module. The graded annihilator $\operatorname{gr-ann}_{R[x, f]} H$ of $H$ is defined in [15, 1.5] and is the largest graded two-sided ideal of $R[x, f]$ that annihilates $H$. We shall use $\mathcal{G}(H)$ (or $\mathcal{G}_{R[x, f]}(H)$ when it is desirable to emphasize the ring $R$ ) to denote the set of all graded annihilators of $R[x, f]$-submodules of $H$.

Recall from [15, 1.5] that an $R[x, f]$-submodule of $H$ is said to be a special annihilator submodule of $H$ if it has the form $\operatorname{ann}_{H}(\mathfrak{B})$ for some graded two-sided ideal $\mathfrak{B}$ of $R[x, f]$. As in [15], we shall use $\mathcal{A}(H)$ to denote the set of special annihilator submodules of $H$, although we shall occasionally expand this notation to $\mathcal{A}_{R[x, f]}(H)$ when it is desirable to specify $R$.

A basic technique from [15] is provided by the following.

1.2. Lemma ([15, Lemma $1.7(\mathrm{v})])$. Let $H$ be a left $R[x, f]$-module. Then the map $\Gamma: \mathcal{A}(H) \longrightarrow \mathcal{G}(H)$ given by

$$
\Gamma(N)=\operatorname{gr}^{-a n n}{ }_{R[x, f]} N \quad \text { for all } N \in \mathcal{A}(H)
$$

is an order-reversing bijection. Its inverse $\Gamma^{-1}: \mathcal{G}(H) \longrightarrow \mathcal{A}(H)$, also orderreversing, is given by

$$
\Gamma^{-1}(\mathfrak{B})=\operatorname{ann}_{H} \mathfrak{B} \quad \text { for all } \mathfrak{B} \in \mathcal{G}(H) .
$$

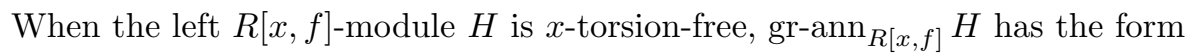

$$
\mathfrak{b} R[x, f]=\bigoplus_{n \in \mathbb{N}_{0}} \mathfrak{b} x^{n}
$$

for some radical ideal $\mathfrak{b}$ of $R$ (by [15, Lemma 1.9]), and, in that case, we write $\mathcal{I}(H)$ for the set of (necessarily radical) ideals $\mathfrak{c}$ of $R$ for which there is an $R[x, f]$ submodule $N$ of $H$ such that gr-ann $\operatorname{six}_{R]} N=\mathfrak{c} R[x, f]$; in these circumstances, the members of $\mathcal{I}(H)$ are referred to as the $H$-special $R$-ideals, and we have $\mathcal{G}_{R[x, f]}(H)=\{\mathfrak{b} R[x, f]: \mathfrak{b} \in \mathcal{I}(H)\}$. Lemma 1.2, in this special case, leads to the following.

1.3. Proposition ([15, Proposition 1.11]). Let $G$ be an $x$-torsion-free left $R[x, f]$ module.

There is an order-reversing bijection $\Delta: \mathcal{A}(G) \longrightarrow \mathcal{I}(G)$ given by

$$
\Delta(N)=\left(\operatorname{gr}^{-a_{n}}{ }_{R[x, f]} N\right) \cap R=\left(0:_{R} N\right) \quad \text { for all } N \in \mathcal{A}(G) .
$$

The inverse $\Delta^{-1}: \mathcal{I}(G) \longrightarrow \mathcal{A}(G)$, also order-reversing, is given by

$$
\left.\Delta^{-1}(\mathfrak{b})=\operatorname{ann}_{G}(\mathfrak{b} R[x, f])\right) \quad \text { for all } \mathfrak{b} \in \mathcal{I}(G) .
$$

The following main result from [15] will also play a key rôle in this paper.

1.4. Theorem ([15, Corollary 3.11]). Let $G$ be an $x$-torsion-free left $R[x, f]$-module. Suppose that $G$ is either Artinian or Noetherian as an $R$-module. Then the set $\mathcal{I}(G)$ of $G$-special R-ideals is finite. 
In [15, §4], the author applied Theorem 1.4 in the case where $(R, \mathfrak{m})$ is an $F$ injective Gorenstein local ring of positive dimension $d$, to the 'top' local cohomology module $H:=H_{\mathfrak{m}}^{d}(R)$ of $R$. (It is worth pointing out that a Gorenstein local ring of characteristic $p$ is $F$-injective if and only if it is $F$-pure.) The statement that $R$ is $F$-injective implies that $H$, with its natural structure as a left $R[x, f]$-module, is $x$-torsion-free, and this implies that $R$ must be reduced. Note also that, in this case, $H_{\mathfrak{m}}^{d}(R) \cong E_{R}(R / \mathfrak{m})$. Theorem 1.4 yields the finite set $\mathcal{I}(H)$ of radical ideals of $R$. Let $\mathfrak{b}$ denote the smallest ideal of positive height in $\mathcal{I}(H)$ (interpret ht $R$ as $\infty)$. In [15, Corollary 4.7] it was shown that, if $c$ is any element of $\mathfrak{b} \cap R^{\circ}$, then $c$ is a test element for $R$, and that $\mathfrak{b}$ is the test ideal $\tau(R)$ of $R$ (that is (in this case), the ideal of $R$ generated by all test elements of $R$ ). These results were obtained without the assumption that $R$ is excellent.

In [16, the author generalized the above-described results of [15, Corollary 4.7] to the case where $(R, \mathfrak{m})$ is local and $E:=E_{R}(R / \mathfrak{m})$ carries a structure of an $x$-torsion-free left $R[x, f]$-module. In this situation, Theorem 1.4 again yields the finite set $\mathcal{I}(E)$ of radical ideals of $R$. One of the main results of [16 is that, if $\mathfrak{b}$ is the smallest ideal of positive height in $\mathcal{I}(E)$, then each element of $\mathfrak{b} \cap R^{\circ}$ is a test element for modules for $R$.

In the case when the local ring $(R, \mathfrak{m})$ is $F$-pure (and $E$ is as above), the graded left $R[x, f]$-module $R[x, f] \otimes_{R} E$ is $x$-torsion-free; in this paper, we shall use a technique similar to that of [16, Theorem 3.5] to show that $\mathcal{I}\left(R[x, f] \otimes_{R} E\right)$ is a finite set, and this will enable us to draw conclusions about tight closure test elements.

1.5. Notation. The notation introduced in the Introduction will be maintained. The symbols $\mathfrak{a}$ and $\mathfrak{b}$ will always denote ideals of $R$.

For $n \in \mathbb{Z}$, we shall denote the $n$th component of a $\mathbb{Z}$-graded left $R[x, f]$-module $G$ by $G_{n}$. If $\phi: L \longrightarrow M$ is a homogeneous homomorphism of $\mathbb{Z}$-graded left $R[x, f]$ modules (of degree 0), then the notation $\phi=\bigoplus_{n \in \mathbb{Z}} \phi_{n}: \bigoplus_{n \in \mathbb{Z}} L_{n} \longrightarrow \bigoplus_{n \in \mathbb{Z}} M_{n}$ will indicate that $\phi_{n}: L_{n} \longrightarrow M_{n}$ is the restriction of $\phi$ to $L_{n}$ (for all $n \in \mathbb{Z}$ ). As in [16. Notation 1.1], we denote the $n$th shift functor on the category of $\mathbb{Z}$-graded left $R[x, f]$-modules and homogeneous homomorphisms (of degree 0 ) by $(\bullet)(n)$. Note that the identity functor on this category is the 0th shift functor.

1.6. Remark. Note that $R x$, which is a left $R$-submodule of $R[x, f]$, has a structure of a right $R$-module via $f$, that is, $r x r^{\prime}=r^{\prime p} r x$ for all $r, r^{\prime} \in R$. Note also that, for a given $R$-module $M$, there is a bijective correspondence between the set of structures as a left $R[x, f]$-module on $M$ (extending its $R$-module structure) and the set

$$
\operatorname{Hom}_{R}\left(R x \otimes_{R} M, M\right),
$$

under which such a structure corresponds to the $R$-homomorphism $\phi$ for which

$$
\phi(r x \otimes m)=r x m \quad \text { for all } r \in R \text { and } m \in M .
$$

Furthermore, given an $R$-homomorphism $\theta: R x \otimes_{R} M \longrightarrow M$, the corresponding left $R[x, f]$-module structure on $M$ is such that $x m=\theta(x \otimes m)$ for all $m \in M$.

We shall make several uses of the following lemma of M. Hochster and J. L. Roberts. 
1.7. Lemma (M. Hochster and J. L. Roberts [7, Lemma 6.2 and Corollary 6.13]). Assume that $R$ is F-pure. Then, for each $\mathfrak{p} \in \operatorname{Spec}(R)$,

(i) the localization $R_{\mathfrak{p}}$ is F-pure, and

(ii) the completion $\widehat{R_{\mathfrak{p}}}$ of $R_{\mathfrak{p}}$ is F-pure.

We shall also need the following result due to R. Fedder.

1.8. Theorem (R. Fedder [3, Theorem 1.12]). Suppose that $R=S / \mathfrak{a}$, where $(S, \mathfrak{n})$ is a regular local ring of characteristic $p$ and $\mathfrak{a}$ is a proper ideal of $S$. Then $R$ is $F$-pure if and only if $\left(\mathfrak{a}^{[p]}: \mathfrak{a}\right) \nsubseteq \mathfrak{n}^{[p]}$.

\section{Homomorphic images of Regular local Rings of CharaCteristic $p$}

Suppose that $(R, \mathfrak{m})$ is local. Recall that $E_{R}(R / \mathfrak{m})$ has a natural structure as a module over the completion $\widehat{R}$ of $R$. Therefore, a structure as a left $R[x, f]$-module on $E_{R}(R / \mathfrak{m})$ induces, in a unique way, a structure as a left $\widehat{R}[x, f]$-module on it extending its $R[x, f]$-module structure. As an $\widehat{R}$-module, $E_{R}(R / \mathfrak{m}) \cong E_{\widehat{R}}(\widehat{R} / \mathfrak{m} \widehat{R})$, and I. S. Cohen's Structure Theorem for complete local rings ensures that $\widehat{R}$ is a homomorphic image of a complete regular local ring of characteristic $p$.

With these considerations in mind, we are going, in this section, to consider possible left $R[x, f]$-module structures on $E_{R}(R / \mathfrak{m})$ when $R$ is a homomorphic image of a regular local ring of characteristic $p$.

2.1. Notation. Throughout this section, we shall assume that $R=S / \mathfrak{a}$, where $S$ is a regular local ring of characteristic $p$ and $\mathfrak{a}$ is a proper, non-zero ideal of $S$. For $s \in S$, we shall denote the natural image of $s$ in $R$ by $\bar{s}$. We shall only assume that $S$ is complete when this is explicitly stated. We shall use $\mathfrak{n}$ to denote the maximal ideal of $S$, so that $\mathfrak{m}:=\mathfrak{n} / \mathfrak{a}$ is the maximal ideal of $R$. Set $E:=E_{S}(S / \mathfrak{n})$, and note that $\left(0:_{E} \mathfrak{a}\right) \cong E_{R}(R / \mathfrak{m})$ as $R$-modules (by [2, 10.1.15], for example). We shall interpret $\left(0:_{E} \mathfrak{a}\right)$ as $E_{R}(R / \mathfrak{m})$.

Observe that $R[x, f]$ is a homomorphic image of $S[x, f]$ under a homomorphism that extends the natural surjective homomorphism from $S$ to $R$ and maps the indeterminate $x$ to $x$. Let $y$ be a new indeterminate. Since $E \cong H_{\mathfrak{n}}^{\operatorname{dim} S}(S)$, the $S$-module $E$ has a natural structure as a left $S[y, f]$-module (as described in, for example, [15, Reminder 4.1]). For any element $u \in S$, it is easy to see (for example, by use of [11, Lemma 1.3]) that we can endow $E$ with a structure of a left $S[x, f]$ module under which $x e=u y e$ for all $e \in E$. A recurring theme of this section is the idea of trying to choose a $u$ as above in such a way that $\left(0:_{E} \mathfrak{a}\right)=E_{R}(R / \mathfrak{m})$ is an $S[x, f]$-submodule of $E$, and so becomes a left $R[x, f]$-module.

For an $S$-submodule $M$ of $E$ and $n \in \mathbb{N}_{0}$, we shall use $S y^{n} M$ to denote the $S$-submodule of $E$ generated by $y^{n} M:=\left\{y^{n} m: m \in M\right\}$. Thus

$$
S y^{n} M=\left\{\sum_{i=1}^{t} s_{i} y^{n} m_{i}: t \in \mathbb{N}, s_{1}, \ldots, s_{t} \in S, m_{1}, \ldots, m_{t} \in M\right\} .
$$

We shall denote $1+p+p^{2}+\cdots+p^{n-1}$, where $n \in \mathbb{N}$, by $\nu_{n}$, and we shall interpret $\nu_{0}$ as 0 . Note that

(i) $p \nu_{n}<1+p \nu_{n}=\nu_{n+1}$,

(ii) $(1-p) \nu_{n}=1-p^{n}$, and

(iii) $\nu_{1}=1$. 
2.2. Remark. We use the notation of $₫ 2.1$ It follows from G. Lyubeznik and K. E. Smith [13, Example 3.7] that, when $S$ is complete, each $S[x, f]$-module structure on $E$ is such that there exists $u \in S$ for which $x e=u y e$ for all $e \in E$.

2.3. Lemma. We use the notation of 2.1, Let $n \in \mathbb{N}$. Then $\left(0:_{S} S y^{n}\left(0:_{E} \mathfrak{a}\right)\right)=$ $\mathfrak{a}^{\left[p^{n}\right]}$.

Proof. Since $S$ is regular, the right $S$-module $S y^{n}$ is flat, and so the homomorphism (of left $S$-modules) $S y^{n} \otimes_{S}(0: E \mathfrak{a}) \longrightarrow S y^{n} \otimes_{S} E$ induced by the inclusion map is injective. However, by [15, Remark 4.2(iii)], the map $\delta_{n}: S y^{n} \otimes_{S} E \longrightarrow E$ for which

$$
\delta_{n}\left(s y^{n} \otimes e\right)=s y^{n} e \quad \text { for all } s \in S \text { and } e \in E
$$

is an $S$-isomorphism. There is therefore a commutative diagram

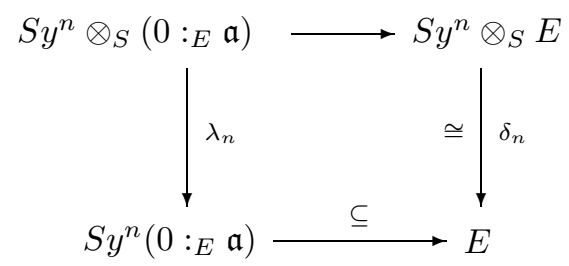

of $S$-modules and $S$-homomorphisms in which the upper horizontal homomorphism is induced by inclusion and the $S$-homomorphism $\lambda_{n}: S y^{n} \otimes_{S}\left(0::_{E} \mathfrak{a}\right) \longrightarrow$ $S y^{n}\left(0:_{E} \mathfrak{a}\right)$ is such that $\lambda_{n}\left(s y^{n} \otimes e\right)=s y^{n} e$ for all $s \in S$ and $e \in\left(0:_{E} \mathfrak{a}\right)$. It follows that $\lambda_{n}$ is an isomorphism, and so the annihilator of $S y^{n}\left(0:_{E} \mathfrak{a}\right)$ is equal to the annihilator of $S y^{n} \otimes_{S}\left(0:_{E} \mathfrak{a}\right)$. However, since the right $S$-module $S y^{n}$ is flat, there are $S$-isomorphisms

$$
\begin{aligned}
S y^{n} \otimes_{S}\left(0:_{E} \mathfrak{a}\right) & \cong S y^{n} \otimes_{S} \operatorname{Hom}_{S}(S / \mathfrak{a}, E) \\
& \cong \operatorname{Hom}_{S}\left(S y^{n} \otimes_{S} S / \mathfrak{a}, S y^{n} \otimes_{S} E\right) \\
& \cong \operatorname{Hom}_{S}\left(S / \mathfrak{a}^{\left[p^{n}\right]}, E\right) .
\end{aligned}
$$

(We have used [14, Theorem 7.11] to obtain the second isomorphism here.) But (even though $S$ might not be complete), an $S$-module and its Matlis dual have equal annihilators. Therefore

$$
\left(0:_{S} S y^{n}\left(0:_{E} \mathfrak{a}\right)\right)=\left(0:_{S} \operatorname{Hom}_{S}\left(S / \mathfrak{a}^{\left[p^{n}\right]}, E\right)\right)=\left(0:_{S} S / \mathfrak{a}^{\left[p^{n}\right]}\right)=\mathfrak{a}^{\left[p^{n}\right]}
$$

2.4. Lemma. We use the notation of $₫ 2.1$. Let $u \in S$. Put the left $S[x, f]$-module structure on $E$ for which $x e=$ uye for all $e \in E$. Then $\left(0:_{E} \mathfrak{a}\right)$ is an $S[x, f]$ submodule of $E$ if and only if $u \in\left(\mathfrak{a}^{[p]}: \mathfrak{a}\right)$.

When this condition is satisfied, $E_{R}(R / \mathfrak{m})$ is a left $R[x, f]$-module with $x e=$ uye for all $e \in\left(0:_{E} \mathfrak{a}\right)=E_{R}(R / \mathfrak{m})$ and $r e=$ se for any $r \in R$ and $s \in S$ for which $\bar{s}=r$.

Note. In the special case of Lemma 2.4 in which $S$ is complete, the results of the lemma can be approached by means of the techniques developed by M. Katzman in [10, $\S \S 3,4]$. However, we now have applications in mind where the full generality of Lemma 2.4 will be required. 
Proof. $(\Leftarrow)$ Let $e \in\left(0:_{E} \mathfrak{a}\right)$. We must show that $x e=$ uye $\in\left(0:_{E} \mathfrak{a}\right)$. So let $s \in \mathfrak{a}$. Since $u \in\left(\mathfrak{a}^{[p]}: \mathfrak{a}\right)$, there exist $a_{1}, \ldots, a_{t} \in \mathfrak{a}$ and $s_{1}, \ldots, s_{t} \in S$ such that $s u=\sum_{i=1}^{t} s_{i} a_{i}^{p}$. Then

$$
\text { sxe }=\text { suye }=\left(\sum_{i=1}^{t} s_{i} a_{i}^{p}\right) \text { ye }=\sum_{i=1}^{t} s_{i} y a_{i} e=0,
$$

since $a_{i} e=0$ for all $i=1, \ldots, t$. Thus $x e \in\left(0:_{E} \mathfrak{a}\right)$. It follows that $\left(0:_{E} \mathfrak{a}\right)$ is an $S[x, f]$-submodule of $E$.

$(\Rightarrow)$ Assume that $\left(0:_{E} \mathfrak{a}\right)$ is an $S[x, f]$-submodule of $E$, so that suye $=0$ for all $s \in \mathfrak{a}$ and $e \in\left(0:_{E} \mathfrak{a}\right)$. Thus, using Lemma 2.3, we see that

$$
u \mathfrak{a} \subseteq\left(0:_{S} S y\left(0:_{E} \mathfrak{a}\right)\right)=\mathfrak{a}^{[p]} .
$$

Therefore $u \in\left(\mathfrak{a}^{[p]}: \mathfrak{a}\right)$.

The claims in the final paragraph are easy to check.

2.5. Lemma (M. Blickle [1, Proposition 3.36]). We use the notation of 42.1 , Suppose that $S$ is complete, and that $\left(0:_{E} \mathfrak{a}\right)$ has a left $R[x, f]$-module structure that extends its $R$-module structure. Then there exists $u \in\left(\mathfrak{a}^{[p]}: \mathfrak{a}\right)$ such that $x e=$ uye for all $e \in\left(0:_{E} \mathfrak{a}\right)$.

Note. This result is included in M. Blickle's Ph.D. dissertation, but we give a short, self-contained proof for the convenience of the reader.

Proof. There is a natural surjective ring homomorphism $S[x, f] \longrightarrow R[x, f]$ which extends the natural ring homomorphism $S \longrightarrow R$ and maps $x$ to $x$. We can use this to consider $\left(0:_{E} \mathfrak{a}\right)$ as an $S[x, f]$-module. By Remark 1.6, there is therefore an $S$-homomorphism $\delta: S x \otimes_{S}\left(0:_{E} \mathfrak{a}\right) \longrightarrow\left(0:_{E} \mathfrak{a}\right)$ such that $\delta(s x \otimes e)=s x e$ for all $s \in S$ and $e \in(0: E \mathfrak{a})$.

Since $S$ is regular, the right $S$-module $S x$ is flat; therefore, the $S$-homomorphism $j: S x \otimes_{S}(0: E \mathfrak{a}) \longrightarrow S x \otimes_{S} E$ induced by inclusion is injective. As $E$ is injective as an $S$-module, there is an $S$-homomorphism $\delta^{\prime}: S x \otimes_{S} E \longrightarrow E$ which makes the diagram

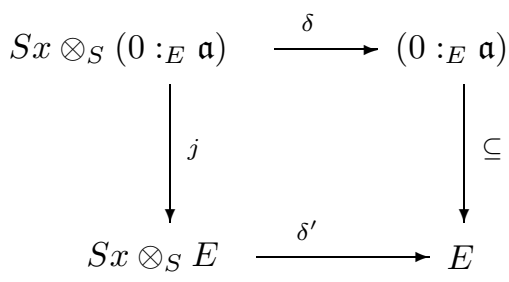

commute.

In view of Remark [1.6] there is therefore a structure of a left $S[x, f]$-module on $E$ which is such that $x e=\delta^{\prime}(x \otimes e)$ for all $e \in E$; this structure extends the $S[x, f]$-module structure on $\left(0:_{E} \mathfrak{a}\right)$.

By Lyubeznik's and Smith's result quoted in Remark 2.2, there exists $u \in S$ for which $x e=u y e$ for all $e \in E$, and, in particular, for all $e \in\left(0:_{E} \mathfrak{a}\right)$. We can now appeal to Lemma 2.4 to see that $u \in\left(\mathfrak{a}^{[p]}: \mathfrak{a}\right)$.

2.6. Remark. We again use the notation of $\$ 2.1$. In the special case in which $S$ is complete, it follows from Matlis duality (see, for example, [18, p. 154]) that each $S$-submodule $M$ of $E$ satisfies $M=\left(0:_{E}\left(0:_{S} M\right)\right)$, and so has the form $\left(0:_{E} \mathfrak{k}\right)$ for a (uniquely determined) ideal $\mathfrak{k}$ of $S$. 
2.7. Proposition. We use the notation of $\$ 2.1$. Let $u \in\left(\mathfrak{a}^{[p]}: \mathfrak{a}\right)$, and put the $S[x, f]$-module structure on $E$ for which $x e=$ uye for all $e \in E$, so that $\left(0:_{E} \mathfrak{a}\right)$ is an $S[x, f]$-submodule of E, by Lemma [2.4. Then

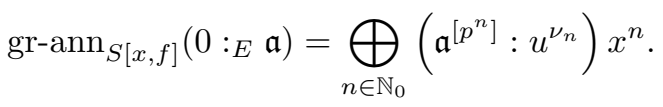

Proof. We can write gr-ann $\left.\operatorname{andx,f]}_{(0}:_{E} \mathfrak{a}\right)=\bigoplus_{n \in \mathbb{N}_{0}} \mathfrak{b}_{n} x^{n}$ for the appropriate ascending chain $\left(\mathfrak{b}_{n}\right)_{n \in \mathbb{N}_{0}}$ of ideals of $S$. In fact,

$$
\mathfrak{b}_{n}=\left\{s \in S: s x^{n} \in \operatorname{ann}_{S[x, f]}(0: E \mathfrak{a})\right\} \quad \text { for all } n \in \mathbb{N}_{0} .
$$

But, for $n \in \mathbb{N}, s \in S$ and $e \in E$,

$$
s x^{n} e=s(u y)^{n} e=s u^{1+p+\cdots+p^{n-1}} y^{n} e=s u^{\nu_{n}} y^{n} e .
$$

Therefore $\mathfrak{b}_{0}=\left(0::_{S}(0: E \mathfrak{a})\right)=\mathfrak{a}=\left(\mathfrak{a}^{\left[p^{0}\right]}: u^{\nu_{0}}\right)$ and, by Lemma 2.3 ,

$$
\mathfrak{b}_{n}=\left\{s \in S: s u^{\nu_{n}} \in\left(0: S S y^{n}\left(0:_{E} \mathfrak{a}\right)\right)=\mathfrak{a}^{\left[p^{n}\right]}\right\}=\left(\mathfrak{a}^{\left[p^{n}\right]}: u^{\nu_{n}}\right) \quad \text { for all } n \in \mathbb{N} .
$$

Next, we record some facts concerning primary decompositions of the ideal $\left(\mathfrak{a}^{[p]}: \mathfrak{a}\right)$.

2.8. Proposition. We use the notation of $\$ 2.1$. Let $\mathfrak{b}_{1}, \ldots, \mathfrak{b}_{t}, \mathfrak{c}$ be ideals of $S$, and let $\mathfrak{a}=\mathfrak{q}_{1} \cap \cdots \cap \mathfrak{q}_{t}$ be a minimal primary decomposition of $\mathfrak{a}$.

(i) We have $\left(\mathfrak{b}_{1} \cap \cdots \cap \mathfrak{b}_{t}\right)^{\left[p^{n}\right]}=\mathfrak{b}_{1}^{\left[p^{n}\right]} \cap \cdots \cap \mathfrak{b}_{t}^{\left[p^{n}\right]}$ for all $n \in \mathbb{N}$.

(ii) If $\mathfrak{q}$ is a $\mathfrak{p}$-primary ideal of $S$, then $\mathfrak{q}^{[p]}$ is also $\mathfrak{p}$-primary.

(iii) If $n \in \mathbb{N}_{0}$, then $\mathfrak{a}^{\left[p^{n}\right]}=\mathfrak{q}_{1}^{\left[p^{n}\right]} \cap \cdots \cap \mathfrak{q}_{t}^{\left[p^{n}\right]}$ is a minimal primary decomposition of $\mathfrak{a}^{\left[p^{n}\right]}$.

(iv) We have $(\mathfrak{a}: \mathfrak{c})^{[p]}=\left(\mathfrak{a}^{[p]}: \mathfrak{c}^{[p]}\right)$ and $\left(\mathfrak{a}^{[p]}: \mathfrak{a}\right) \subseteq\left((\mathfrak{a}: \mathfrak{c})^{[p]}:(\mathfrak{a}: \mathfrak{c})\right)$.

(v) If $\mathfrak{p}$ is an associated prime ideal of $\mathfrak{a}$, then $\left(\mathfrak{a}^{[p]}: \mathfrak{a}\right) \subseteq\left(\mathfrak{p}^{[p]}: \mathfrak{p}\right)$.

(vi) Since $0 \neq \mathfrak{a} \neq S$, we have $\left(\mathfrak{a}^{[p]}: \mathfrak{a}\right) \neq S$. If $\mathfrak{p}_{1}:=\sqrt{\mathfrak{q}_{1}}$ is a minimal prime ideal of $\mathfrak{a}$, then $\mathfrak{p}_{1}$ is a minimal prime ideal of $\left(\mathfrak{a}^{[p]}: \mathfrak{a}\right)$ and the unique $\mathfrak{p}_{1}$-primary component of $\left(\mathfrak{a}^{[p]}: \mathfrak{a}\right)$ is $\left(\mathfrak{q}_{1}^{[p]}: \mathfrak{q}_{1}\right)$.

(vii) If $\mathfrak{q}$ is a $\mathfrak{p}$-primary irreducible ideal of $S$, then $\mathfrak{q}^{[p]}$ is also $\mathfrak{p}$-primary and irreducible.

Proof. (i), (ii), (iii) These are immediate consequences of the fact that the Frobenius homomorphism is a faithfully flat ring homomorphism from $S$ to $S$.

(iv) That $(\mathfrak{a}: \mathfrak{c})^{[p]}=\left(\mathfrak{a}^{[p]}: \mathfrak{c}^{[p]}\right)$ also follows from the faithful flatness of the Frobenius ring homomorphism on $S$. Furthermore, since $\left(\mathfrak{a}^{[p]}: \mathfrak{a}\right)(\mathfrak{a}: \mathfrak{c}) \mathfrak{c} \subseteq \mathfrak{a}^{[p]}$, it is clear that $\left(\mathfrak{a}^{[p]}: \mathfrak{a}\right)(\mathfrak{a}: \mathfrak{c}) \mathfrak{c}^{[p]} \subseteq \mathfrak{a}^{[p]}$, and therefore

$$
\left(\mathfrak{a}^{[p]}: \mathfrak{a}\right)(\mathfrak{a}: \mathfrak{c}) \subseteq\left(\mathfrak{a}^{[p]}: \mathfrak{c}^{[p]}\right)=(\mathfrak{a}: \mathfrak{c})^{[p]} .
$$

(v) This is immediate from part (iv), since there exists $s \in S$ such that $\mathfrak{p}=(\mathfrak{a}: s)$.

(vi) If it were the case that $\mathfrak{a}^{[p]}=\mathfrak{a}$, then we should have $\mathfrak{n} \mathfrak{a}=\mathfrak{a}$, so that $\mathfrak{a}$ would be zero, by Nakayama's Lemma; therefore $\left(\mathfrak{a}^{[p]}: \mathfrak{a}\right) \neq S$.

It follows from part (iii) that

$$
\left(\mathfrak{a}^{[p]}: \mathfrak{a}\right)=\left(\mathfrak{q}_{1}^{[p]} \cap \cdots \cap \mathfrak{q}_{t}^{[p]}: \mathfrak{a}\right)=\left(\mathfrak{q}_{1}^{[p]}: \mathfrak{a}\right) \cap \cdots \cap\left(\mathfrak{q}_{t}^{[p]}: \mathfrak{a}\right),
$$


and from part (ii) that this becomes a primary decomposition of $\left(\mathfrak{a}^{[p]}: \mathfrak{a}\right)$ after terms equal to $S$ are discarded, since, for each $i=1, \ldots, t$, the ideal $\left(\mathfrak{q}_{i}^{[p]}: \mathfrak{a}\right)$ is either $\sqrt{\mathfrak{q}_{i}}$-primary or equal to $S$.

For the remainder of this proof, for convenience, we denote $\mathfrak{p}_{1}$ by $\mathfrak{p}$ and $\mathfrak{q}_{1}$ by $\mathfrak{q}$. Let ${ }^{e}$ and ${ }^{c}$ stand for extension and contraction of ideals with respect to the natural ring homomorphism $S \longrightarrow S_{\mathfrak{p}}$. Then $\left(\mathfrak{q}_{i}^{[p]}: \mathfrak{a}\right)^{e c}=S$ for all $i=2, \ldots, t$, since $\mathfrak{p}$ is a minimal prime ideal of $\mathfrak{a}$; therefore

$$
\left(\mathfrak{a}^{[p]}: \mathfrak{a}\right)^{e c}=\left(\mathfrak{q}^{[p]}: \mathfrak{a}\right)^{e c}=\left(\left(\mathfrak{q}^{[p]}\right)^{e}: \mathfrak{a}^{e}\right)^{c}=\left(\left(\mathfrak{q}^{[p]}\right)^{e}: \mathfrak{q}^{e}\right)^{c}=\left(\mathfrak{q}^{[p]}: \mathfrak{q}\right)^{e c}=\left(\mathfrak{q}^{[p]}: \mathfrak{q}\right)
$$

since $\left(\mathfrak{q}^{[p]}: \mathfrak{q}\right)$ is $\mathfrak{p}$-primary by part (ii) and the first paragraph of the proof of this part (vi).

We can now conclude from the last two paragraphs that $\mathfrak{p}$ is a minimal prime ideal of $\left(\mathfrak{a}^{[p]}: \mathfrak{a}\right)$ and the unique $\mathfrak{p}$-primary component of $\left(\mathfrak{a}^{[p]}: \mathfrak{a}\right)$ is $\left(\mathfrak{q}^{[p]}: \mathfrak{q}\right)$.

(vii) This was established in [17, Remark 8.3(iii)] by use of a result of Huneke and Sharp [9, Proposition 1.5].

2.9. Proposition. We use the notation of 2.1 . Let $\mathfrak{p} \in \operatorname{Spec}(S)$ and let $u \in\left(\mathfrak{p}^{[p]}: \mathfrak{p}\right) \backslash \mathfrak{p}^{[p]}$. Put the $S[x, f]$-module structure on $E$ for which $x e=$ uye for all $e \in E$, so that $\left(0:_{E} \mathfrak{p}\right)$ is an $S[x, f]$-submodule of $E$, by Lemma 2.4 .

Then $\left(\mathfrak{p}^{\left[p^{n}\right]}: u^{\nu_{n}}\right)=\mathfrak{p}$ for all $n \in \mathbb{N}_{0}$, so that $\left(0:_{E} \mathfrak{p}\right)$ is not $x$-torsion.

Proof. The claim that $\left(\mathfrak{p}^{\left[p^{n}\right]}: u^{\nu_{n}}\right)=\mathfrak{p}$ is obvious when $\mathfrak{p}=0$ or $n=0$; we therefore suppose that $\mathfrak{p} \neq 0$ and $n>0$.

By Proposition 2.8 (ii), the Frobenius power $\mathfrak{p}^{[p]}$ is $\mathfrak{p}$-primary; since $u \notin \mathfrak{p}^{[p]}$, the ideal $\left(\mathfrak{p}^{[p]}: u\right)$ is $\mathfrak{p}$-primary, so that $\left(\mathfrak{p}^{[p]}: u\right) \subseteq \mathfrak{p}$. But $u \mathfrak{p} \subseteq \mathfrak{p}^{[p]}$, and so

$$
\mathfrak{p} \subseteq\left(\mathfrak{p}^{[p]}: u\right) \subseteq \mathfrak{p}
$$

Therefore $\left(\mathfrak{p}^{[p]}: u\right)=\mathfrak{p}$ and the desired result has been proved in the case where $n=1$.

Now suppose, inductively, that $n \geq 1$ and we have shown that $\left(\mathfrak{p}^{\left[p^{n}\right]}: u^{\nu_{n}}\right)=\mathfrak{p}$. We can use Proposition 2.8(iv) to deduce that

$$
\mathfrak{p}^{[p]}=\left(\mathfrak{p}^{\left[p^{n}\right]}: u^{\nu_{n}}\right)^{[p]}=\left(\left(\mathfrak{p}^{\left[p^{n}\right]}\right)^{[p]}:\left(u^{\nu_{n}}\right)^{p}\right)=\left(\mathfrak{p}^{\left[p^{n+1}\right]}: u^{\nu_{n} p}\right) .
$$

But $\nu_{n} p+1=\nu_{n+1}$, and so

$$
\left(\mathfrak{p}^{\left[p^{n+1}\right]}: u^{\nu_{n+1}}\right)=\left(\mathfrak{p}^{\left[p^{n+1}\right]}: u^{\nu_{n} p} u\right)=\left(\left(\mathfrak{p}^{\left[p^{n+1}\right]}: u^{\nu_{n} p}\right): u\right)=\left(\mathfrak{p}^{[p]}: u\right)=\mathfrak{p}
$$

by the case where $n=1$. This completes the inductive step.

It now follows from Proposition 2.7 that gr-ann ${ }_{S[x, f]}\left(0:_{E} \mathfrak{p}\right)=\bigoplus_{n \in \mathbb{N}_{0}} \mathfrak{p} x^{n}$. Note that $\left(0:_{E} \mathfrak{p}\right)$ is Artinian as an $S$-module. We now see from the Hartshorne-SpeiserLyubeznik Theorem (see G. Lyubeznik [12, Proposition 4.4] and R. Hartshorne and R. Speiser [4, Proposition 1.11]) that if $\left(0:_{E} \mathfrak{p}\right)$ were $x$-torsion, then it would be annihilated by $x^{h}$ for some $h \in \mathbb{N}$. As this is not the case, $\left(0:_{E} \mathfrak{p}\right)$ is not $x$ torsion.

2.10. Proposition. Again use the notation of 2.1 . Assume that $S$ is complete and the (complete) local ring $R=S / \mathfrak{a}$ is Gorenstein. Then $\left(0:_{E} \mathfrak{a}\right)=E_{R}(R / \mathfrak{m}) \cong$ $H_{\mathfrak{m}}^{\operatorname{dim} R}(R)$, and so has a natural structure as a left $R[x, f]$-module. By Lemma 2.5, there exists $u \in\left(\mathfrak{a}^{[p]}: \mathfrak{a}\right)$ such that, under this natural structure, xe $=$ uye for all $e \in\left(0:_{E} \mathfrak{a}\right)$. Then $\left(\mathfrak{a}^{[p]}: \mathfrak{a}\right)=\mathfrak{a}^{[p]}+S u$. 
Proof. Let $z$ be a new variable. It follows from Lyubeznik and Smith [13, Example $3.7]$ that, since $R$ is complete and Gorenstein, each $R[z, f]$-module structure on $\left(0:_{E} \mathfrak{a}\right)$ is such that there exists $w^{\prime} \in S$ for which $z e=\overline{w^{\prime}} x e$ for all $e \in\left(0:_{E} \mathfrak{a}\right)$.

Let $v \in\left(\mathfrak{a}^{[p]}: \mathfrak{a}\right)$. By Lemma 2.4, there is a left $R[z, f]$-module structure on $\left(0:_{E} \mathfrak{a}\right)$ for which $z e=v y e$ for all $e \in\left(0:_{E} \mathfrak{a}\right)$. By the first paragraph of this

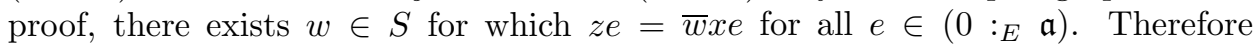
$v y e=z e=w x e=w u y e$ for all $e \in\left(0:_{E} \mathfrak{a}\right)$, and $v-w u$ annihilates $S y\left(0:_{E} \mathfrak{a}\right)$, so that $v-w u \in \mathfrak{a}^{[p]}$ by Lemma 2.3. Therefore $\left(\mathfrak{a}^{[p]}: \mathfrak{a}\right) \subseteq \mathfrak{a}^{[p]}+S u$; the reverse inclusion is obvious.

2.11. Corollary. Once again, we use the notation of 2.1 . The minimum number of generators of the $S$-module $\left(\mathfrak{a}^{[p]}: \mathfrak{a}\right) / \mathfrak{a}^{[p]}$ is equal to the minimum number of generators of the $\widehat{S}$-module $\left((\mathfrak{a} \widehat{S})^{[p]}: \mathfrak{a} \widehat{S}\right) /(\mathfrak{a} \widehat{S})^{[p]}$.

Consequently, if the local ring $R=S / \mathfrak{a}$ is Gorenstein, then $\left(\mathfrak{a}^{[p]}: \mathfrak{a}\right) / \mathfrak{a}^{[p]}$ is a cyclic $S$-module.

Proof. Let $t$ denote the minimal number of generators of the $S$-module $\left(\mathfrak{a}^{[p]}: \mathfrak{a}\right) / \mathfrak{a}^{[p]}$; then $t$ is equal to the dimension of $\left(\mathfrak{a}^{[p]}: \mathfrak{a}\right) /\left(\mathfrak{n}\left(\mathfrak{a}^{[p]}: \mathfrak{a}\right)+\mathfrak{a}^{[p]}\right)$ as a vector space over $S / \mathfrak{n}$. As $\widehat{S}$ is faithfully flat over $S$, it is straightforward to check that this dimension is equal to the dimension, as a vector space over $\widehat{S} / \mathfrak{n} \widehat{S}$, of

$$
\left((\mathfrak{a} \widehat{S})^{[p]}: \mathfrak{a} \widehat{S}\right) /\left(\mathfrak{n} \widehat{S}\left((\mathfrak{a} \widehat{S})^{[p]}: \mathfrak{a} \widehat{S}\right)+(\mathfrak{a} \widehat{S})^{[p]}\right) .
$$

Therefore the minimum number of generators of the $\widehat{S}$-module

$$
\left((\mathfrak{a} \widehat{S})^{[p]}: \mathfrak{a} \widehat{S}\right) /(\mathfrak{a} \widehat{S})^{[p]}
$$

is also equal to $t$.

Note that $\widehat{R}=\widehat{S} / \mathfrak{a} \widehat{S}$ is Gorenstein if and only if $R$ is Gorenstein. Therefore, in order to prove the claim in the final paragraph of the corollary, it is sufficient for us to do so in the case where $S$ is complete, and, in that case, the claim is immediate from Proposition 2.10.

2.12. Proposition. Use the notation of 42.1. Suppose that $u_{1}, u_{2} \in S$ are such that

$$
\left(\mathfrak{a}^{[p]}: \mathfrak{a}\right)=\mathfrak{a}^{[p]}+S u_{1}=\mathfrak{a}^{[p]}+S u_{2} .
$$

Bearing in mind Lemma 2.4. let $D_{i}$, for $i=1,2$, denote $\left(0:_{E} \mathfrak{a}\right)$ endowed with the left $R[x, f]$-module structure that (extends its $R$-module structure and) is such that $x e=u_{i}$ ye for all $e \in\left(0:_{E} \mathfrak{a}\right)$. Then a subset of $\left(0:_{E} \mathfrak{a}\right)$ is an $R[x, f]$-submodule of $D_{1}$ if and only if it is an $R[x, f]$-submodule of $D_{2}$.

Proof. There exist $t \in \mathfrak{a}^{[p]}$ and $s \in S$ such that $u_{1}=t+s u_{2}$, and $a_{1}, \ldots, a_{h} \in \mathfrak{a}$ and $s_{1}, \ldots, s_{h} \in S$ such that $t=\sum_{i=1}^{h} s_{i} a_{i}^{p}$; therefore, for $e \in\left(0:_{E} \mathfrak{a}\right)$, we have

$$
\text { tye }=\left(\sum_{i=1}^{h} s_{i} a_{i}^{p}\right) \text { ye }=\sum_{i=1}^{h} s_{i} a_{i}^{p} y e=\sum_{i=1}^{h} s_{i} y a_{i} e=0 .
$$

Hence $u_{1} y e=s u_{2} y e$ for all $e \in\left(0:_{E} \mathfrak{a}\right)$, so that every $R[x, f]$-submodule of $D_{2}$ is an $R[x, f]$-submodule of $D_{1}$. We can similarly prove that every $R[x, f]$-submodule of $D_{1}$ is an $R[x, f]$-submodule of $D_{2}$. 
2.13. Corollary. Again use the notation of $₫ 2.1$, so that $(R, \mathfrak{m})$ is local and equal to the homomorphic image $S / \mathfrak{a}$ of the regular local ring $S$.

Suppose that $u \in S$ is such that $\left(\mathfrak{a}^{[p]}: \mathfrak{a}\right)=\mathfrak{a}^{[p]}+S u$. Bearing in mind Lemma 2.4, let $D$ denote $\left(0:_{E} \mathfrak{a}\right)$ endowed with the left $R[x, f]$-module structure that (extends its $R$-module structure and) is such that $x e=$ uye for all $e \in\left(0::_{E} \mathfrak{a}\right)$. If $R$ is regular, then $D$ is a simple $R[x, f]$-module.

Proof. Let $d$ denote $\operatorname{dim} R$. Since $E_{R}(R / \mathfrak{m})$ has a natural structure as a module over the completion $\widehat{R}$ of $R$, the specified structure as a left $R[x, f]$-module on $E_{R}(R / \mathfrak{m})$ induces, in a unique way, a structure as a left $\widehat{R}[x, f]$-module on it extending its $R[x, f]$-module structure. Note that $\widehat{R}=\widehat{S} / \mathfrak{a} \widehat{S}$, that $\left((\mathfrak{a} \widehat{S})^{[p]}:_{\widehat{S}} \mathfrak{a} \widehat{S}\right)=(\mathfrak{a} \widehat{S})^{[p]}+\widehat{S} u$, and that

$$
\left(0:_{E} \mathfrak{a}\right)=\left(0:_{E} \mathfrak{a} \widehat{S}\right)=E_{\widehat{R}}(\widehat{R} / \mathfrak{m} \widehat{R}) .
$$

Because $\widehat{R}$ is regular, $\left(0:_{E} \mathfrak{a} \widehat{S}\right) \cong H_{\mathfrak{m} \widehat{R}}^{d}(\widehat{R})$ as $\widehat{R}$-modules. The natural left $\widehat{R}[x, f]$-module structure on this can, by Lemma 2.5 be described by some $\widehat{v} \in$ $\left((\mathfrak{a} \widehat{S})^{[p]}: \widehat{S} \mathfrak{a} \widehat{S}\right)$, in the sense that, under this natural structure, $x e=\widehat{v} y e$ for all $e \in\left(0:_{E} \mathfrak{a} \widehat{R}\right)$. By Proposition 2.10, $\left((\mathfrak{a} \widehat{S})^{[p]}: \widehat{S} \mathfrak{a} \widehat{S}\right)=(\mathfrak{a} \widehat{S})^{[p]}+\widehat{S} \widehat{v}$.

Since a subset of $\left(0:_{E} \mathfrak{a} \widehat{S}\right)$ is an $R$-submodule if and only if it is an $\widehat{R}$-submodule, it follows from Proposition 2.12 that $D$ is simple as a left $R[x, f]$-module if and only if $H_{\mathfrak{m} \widehat{R}}^{d}(\widehat{R})$ is simple as a left $\widehat{R}[x, f]$-module under its natural structure. But, as $\widehat{R}$ is excellent (as it is complete) and regular, this is immediate from a result of K. E. Smith [19, Theorem 2.6].

\section{Applications to F-pure local rings}

In [16, Theorem 3.5], the author showed that, if $(R, \mathfrak{m})$ is local and the $R$-module structure on $E_{R}(R / \mathfrak{m})$ can be extended to an $x$-torsion-free left $R[x, f]$-module structure, then $R$ is $F$-pure. The first two results in this section establish the converse.

3.1. Proposition. Use the notation of 2.1, Suppose that $R$ is F-pure, so that, by Fedder's Theorem 1.8 , there exists $u \in\left(\mathfrak{a}^{[p]}: \mathfrak{a}\right) \backslash \mathfrak{n}^{[p]}$. Put the left $S[x, f]$-module structure on $E$ for which $x e=$ uye for all $e \in E$, so that $\left(0:_{E} \mathfrak{a}\right)=E_{R}(R / \mathfrak{m})$ is, by Lemma 2.4, an $S[x, f]$-submodule of $E$ which inherits a left $R[x, f]$-module structure (that extends its $R$-module structure).

With this left $R[x, f]$-module structure, $E_{R}(R / \mathfrak{m})$ is $x$-torsion-free.

Proof. As in the first paragraph of the proof of Corollary 2.13, we can extend the specified $R[x, f]$-module structure on $E_{R}(R / \mathfrak{m})$ to an $\widehat{R}[x, f]$-module structure, in a unique way: note that $\widehat{R}$ is $F$-pure, by Lemma 1.7 and $u \in\left((\mathfrak{a} \widehat{S})^{[p]}: \widehat{S} \mathfrak{a} \widehat{S}\right) \backslash(\mathfrak{n} \widehat{S})^{[p]}$. Thus we can, and do, assume that $R$ and $S$ are complete.

It now follows from Remark 2.6 that, if $\mathfrak{b}$ denotes $\left(0:_{S} \Gamma_{x}\left(E_{R}(R / \mathfrak{m})\right)\right)$, then $\Gamma_{x}\left(E_{R}(R / \mathfrak{m})\right)=\left(0:_{E} \mathfrak{b}\right)$. We suppose that $\mathfrak{b}$ is a proper ideal of $S$ and seek a contradiction. Since $\Gamma_{x}\left(E_{R}(R / \mathfrak{m})\right)$ is an $S[x, f]$-submodule of $E$, we conclude from Lemma 2.4 that $u \in\left(\mathfrak{b}^{[p]}: \mathfrak{b}\right)$. Let $\mathfrak{p}$ be a minimal prime ideal of $\mathfrak{b}$. Then it follows from Proposition $2.8(\mathrm{v})$ that $u \in\left(\mathfrak{p}^{[p]}: \mathfrak{p}\right)$, so that $\left(0:_{E} \mathfrak{p}\right)$ is an $S[x, f]$-submodule of $E$, by Lemma 2.4. Since $\mathfrak{p} \supseteq \mathfrak{b}$, we have $\left(0:_{E} \mathfrak{p}\right) \subseteq\left(0:_{E} \mathfrak{b}\right)=\Gamma_{x}\left(E_{R}(R / \mathfrak{m})\right)$; therefore $\left(0:_{E} \mathfrak{p}\right)$ is an $x$-torsion $S[x, f]$-submodule of $E$. 
We can now use Proposition 2.9 to deduce that $u \in \mathfrak{p}^{[p]}$, so that, since $\mathfrak{p} \subseteq \mathfrak{n}$, we must have $u \in \mathfrak{n}^{[p]}$. This contradicts the choice of $u$. Hence $\mathfrak{b}=S$ and $E_{R}(R / \mathfrak{m})$ is an $x$-torsion-free left $R[x, f]$-module.

3.2. Theorem. Suppose that $(R, \mathfrak{m})$ is local. Then $R$ is F-pure if and only if the $R$-module structure on $E_{R}(R / \mathfrak{m})$ can be extended to an $x$-torsion-free left $R[x, f]$ module structure.

Proof. $(\Leftarrow)$ This was proved in [16, Theorem 3.5].

$\Rightarrow$ ) Assume that $R$ is $F$-pure; note that, by Lemma 1.7, this implies that the completion $\widehat{R}$ of $R$ is $F$-pure. Since $E_{R}(R / \mathfrak{m})$ has a natural structure as a module over $\widehat{R}$, and as there is an $\widehat{R}$-isomorphism $E_{R}(R / \mathfrak{m}) \cong E_{\widehat{R}}(\widehat{R} / \mathfrak{m} \widehat{R})$, we can, and do, assume that $R$ is complete.

We now appeal to Cohen's Structure Theorem for complete local rings containing a subfield and write $R=S / \mathfrak{a}$, where $S$ is a regular local ring of characteristic $p$ and $\mathfrak{a}$ is a proper, non-zero ideal of $S$. It now follows from Proposition 3.1 that the $R$-module structure on $E_{R}(R / \mathfrak{m})$ can be extended to an $x$-torsion-free left $R[x, f]$ module structure.

3.3. Corollary. Suppose that $(R, \mathfrak{m})$ is local and F-pure. Then $R$ (is reduced and) has a tight closure test element for modules.

Note. It should be noted that, in Corollary 3.3 , we have not assumed that $R$ is excellent.

Proof. By Theorem 3.2, the $R$-module structure on $E_{R}(R / \mathfrak{m})$ can be extended to an $x$-torsion-free left $R[x, f]$-module structure. It now follows from [16, Theorem $3.5(\mathrm{i})$ ] that $R$ (is reduced and) has a tight closure test element for modules.

One of the main aims of this paper is to prove that if $R$ is (not necessarily local but) excellent and $F$-pure, then $R$ has a big tight closure test element. In fact, we shall show in $\$ 4$ that if $c \in R^{\circ}$ is such that $R_{c}$ is regular, then $c$ itself is a big test element for $R$. In this section, we shall concentrate on the case where $(R, \mathfrak{m})$ is local (and $F$-pure and excellent).

3.4. Remarks. Suppose that $R$ is excellent and reduced.

(i) There exists $c \in R^{\circ}$ such that $R_{c}$ is regular. This can be proved easily by prime avoidance arguments, based on the facts that

$$
\operatorname{Reg}(R):=\left\{\mathfrak{p} \in \operatorname{Spec}(R): R_{\mathfrak{p}} \text { is a regular local ring }\right\}
$$

is open in $\operatorname{Spec}(R)$, and, for each minimal prime ideal $\mathfrak{q}$ of $R$, the localization $R_{\mathfrak{q}}$ is a field and so regular.

(ii) Since $R$ is reduced and excellent, the concepts of test element for modules and test element for ideals for $R$ coincide; see [5, Discussion (8.6) and Proposition (8.15)]. If $(R, \mathfrak{m})$ is local and $c \in R^{\circ}$ is a test element for the completion $\widehat{R}$ of $R$, then $c$ is a test element for $R$.

3.5. Lemma. Use the notation of \2.1, and suppose that $R$ is F-pure. Let $t$ denote the minimum number of generators of the $S$-module $T:=\left(\mathfrak{a}^{[p]}: \mathfrak{a}\right) / \mathfrak{a}{ }^{[p]}$. Then it is possible to find $t$ elements $u_{1}, \ldots, u_{t} \in\left(\mathfrak{a}^{[p]}: \mathfrak{a}\right) \backslash \mathfrak{n}^{[p]}$ whose natural images in $T$ generate $T$. 
Proof. Suppose, inductively, that $j \in \mathbb{N}_{0}$ is such that $j<t$ and we have constructed $u_{1}, \ldots, u_{j} \in\left(\mathfrak{a}^{[p]}: \mathfrak{a}\right) \backslash \mathfrak{n}^{[p]}$ whose natural images in $T / \mathfrak{n} T$ are linearly independent over the field $S / \mathfrak{n}$. This is certainly true when $j=0$. Note that $\left(\mathfrak{a}^{[p]}: \mathfrak{a}\right) \nsubseteq \mathfrak{n}^{[p]}$, by Fedder's Theorem 1.8. By ideal avoidance, there exists

$$
u_{j+1} \in\left(\mathfrak{a}^{[p]}: \mathfrak{a}\right) \backslash\left(\mathfrak{n}^{[p]} \cup\left(\mathfrak{n}\left(\mathfrak{a}^{[p]}: \mathfrak{a}\right)+\mathfrak{a}^{[p]}+S u_{1}+\cdots+S u_{j}\right)\right)
$$

(or else the vector space $T / \mathfrak{n} T$ over $S / \mathfrak{n}$ would have dimension less than $t$ ). Then the natural images of $u_{1}, \ldots, u_{j}, u_{j+1}$ in $T / \mathfrak{n} T$ are linearly independent over $S / \mathfrak{n}$. This completes the inductive step.

Hence, by induction, we can construct $u_{1}, \ldots, u_{t} \in\left(\mathfrak{a}^{[p]}: \mathfrak{a}\right) \backslash \mathfrak{n}^{[p]}$ whose natural images in $T / \mathfrak{n} T$ are linearly independent over the field $S / \mathfrak{n}$; the natural images of $u_{1}, \ldots, u_{t}$ in $T$ generate this $S$-module.

3.6. Proposition. Use the notation of 92.1 , and suppose that $R$ is F-pure and that $S($ and $R)$ are complete. Use Lemma 3.5 to find $u_{1}, \ldots, u_{t} \in\left(\mathfrak{a}^{[p]}: \mathfrak{a}\right) \backslash \mathfrak{n}^{[p]}$ such that their natural images in $\left(\mathfrak{a}^{[p]}: \mathfrak{a}\right) / \mathfrak{a}^{[p]}$ form a generating set for this $S$ module. Bearing in mind Lemma 2.4, for each $i=1, \ldots, t$, let $E_{i}$ denote $\left(0:_{E} \mathfrak{a}\right)=$ $E_{R}(R / \mathfrak{m})$ endowed with the left $R[x, f]$-module structure for which $x e=u_{i}$ ye for all $e \in\left(0:_{E} \mathfrak{a}\right)$, and note that $E_{i}$ is $x$-torsion-free, by Proposition 3.1 ; let $\mathfrak{b}_{i}$ be the unique smallest ideal of positive height in $\mathcal{I}\left(E_{i}\right)$.

Set $\mathfrak{b}=\mathfrak{b}_{1}+\cdots+\mathfrak{b}_{t}$, and let $c \in R$ be such that $R_{c}$ is regular. Then $\mathfrak{b} R_{c}=R_{c}$.

Proof. Let $\mathfrak{p} \in \operatorname{Spec}(R)$ be such that $c \notin \mathfrak{p}$. Then $R_{\mathfrak{p}}$, being a localization of $R_{c}$, is regular. Let $\mathfrak{q}$ be the prime ideal of $S$ that contains $\mathfrak{a}$ and is such that $\mathfrak{p}=\mathfrak{q} / \mathfrak{a}$. Note that

$$
\left(\left(\mathfrak{a} S_{\mathfrak{q}}\right)^{[p]}: \mathfrak{a} S_{\mathfrak{q}}\right)=\left(\mathfrak{a} S_{\mathfrak{q}}\right)^{[p]}+\left(u_{1} / 1\right) S_{\mathfrak{q}}+\cdots+\left(u_{t} / 1\right) S_{\mathfrak{q}} .
$$

Since $S_{\mathfrak{q}} / \mathfrak{a} S_{\mathfrak{q}} \cong R_{\mathfrak{p}}$ is a regular local ring, it follows from Corollary 2.11 that the $S_{\mathfrak{q}}$-module $\left(\left(\mathfrak{a} S_{\mathfrak{q}}\right)^{[p]}: \mathfrak{a} S_{\mathfrak{q}}\right) / \mathfrak{a} S_{\mathfrak{q}}^{[p]}$ is cyclic; therefore, there exists $i \in\{1, \ldots, t\}$ such that

$$
\left(\left(\mathfrak{a} S_{\mathfrak{q}}\right)^{[p]}: \mathfrak{a} S_{\mathfrak{q}}\right)=\mathfrak{a} S_{\mathfrak{q}}^{[p]}+\left(u_{i} / 1\right) S_{\mathfrak{q}}
$$

Let $G$ denote the injective envelope, over $R_{\mathfrak{p}}$, of the simple $R_{\mathfrak{p}}$-module, and use Lemma 2.4 in conjunction with the isomorphism $R_{\mathfrak{p}} \cong S_{\mathfrak{q}} / \mathfrak{a} S_{\mathfrak{q}}$ and the element $u_{i} / 1$ to put a left $R_{\mathfrak{p}}[x, f]$-module structure on $G$.

Let $\mathfrak{k}_{i}$ be the ideal of $S$ that contains $\mathfrak{a}$ and is such that $\mathfrak{b}_{i}=\mathfrak{k}_{i} / \mathfrak{a}$. Since $R$ is complete, it follows from [16, Proposition 1.8] that

$$
\left(0::_{E} \mathfrak{k}_{i}\right)=\left(0:_{E_{R}(R / \mathfrak{m})} \mathfrak{b}_{i}\right)=\operatorname{ann}_{E_{i}}\left(\mathfrak{b}_{i} R[x, f]\right),
$$

so that this is an $R[x, f]$-submodule of $E_{i}$. It therefore follows from Lemma 2.4 that $u_{i} \in\left(\left(\mathfrak{k}_{i}\right)^{[p]}: \mathfrak{k}_{i}\right)$. Therefore, in $S_{\mathfrak{q}}$, we have

$$
u_{i} / 1 \in\left(\left(\mathfrak{k}_{i} S_{\mathfrak{q}}\right)^{[p]}: \mathfrak{k}_{i} S_{\mathfrak{q}}\right)
$$

and so $\left(0::_{G} \mathfrak{k}_{i} S_{\mathfrak{q}} / \mathfrak{a} S_{\mathfrak{q}}\right)=\left(\begin{array}{lll}0 & :_{G} & \mathfrak{b}_{i} R_{\mathfrak{p}}\end{array}\right)$ is an $R_{\mathfrak{p}}[x, f]$-submodule of $G$, again by Lemma 2.4. Now $G$ is simple as an $R_{\mathfrak{p}}[x, f]$-module, by Corollary 2.13. Therefore, since a module over a (not necessarily complete) local ring has the same annihilator as its Matlis dual, $\mathfrak{b}_{i} R_{\mathfrak{p}}$ must be 0 or $R_{\mathfrak{p}}$. Since ht $\mathfrak{b}_{i} R_{\mathfrak{p}} \geq 1$, we must have $\mathfrak{b}_{i} R_{\mathfrak{p}}=$ $R_{\mathfrak{p}}$.

Therefore $\mathfrak{b} R_{\mathfrak{p}}=R_{\mathfrak{p}}$. As the latter equation is true for all $\mathfrak{p} \in \operatorname{Spec}(R)$ such that $c \notin \mathfrak{p}$, we see that $\mathfrak{b} R_{c}=R_{c}$. 
3.7. Theorem. Suppose that $(R, \mathfrak{m})$ is local, F-pure and excellent. Let $c \in R^{\circ}$ be such that $R_{c}$ is regular. (By Remark 3.4(i), it is possible to find such a c.) Then $c$ is a test element for both $R$ and $\widehat{R}$.

Proof. By Remark 3.4(ii), it is sufficient to prove that $c$ is a test element for $\widehat{R}$. Note that $\widehat{R}$ is $F$-pure, by Lemma 1.7. Complete local rings are always excellent. Furthermore, the fibre rings of the flat ring homomorphism $R_{c} \longrightarrow \widehat{R}_{c}$ induced by inclusion are rings of fractions of the formal fibres of $R$, and so are regular; it therefore follows that $\widehat{R}_{c}$ is regular. Thus we can, and do, assume for the remainder of this proof that $R$ is complete.

We now appeal to Cohen's Structure Theorem for complete local rings containing a subfield and write $R=S / \mathfrak{a}$, where $S$ is a complete regular local ring of characteristic $p$ and $\mathfrak{a}$ is a proper, non-zero ideal of $S$. This is consistent with the notation of 42.1 , and we shall use that notation for the remainder of this proof.

We now appeal to Proposition 3.6. Use Lemma 3.5 to find

$$
u_{1}, \ldots, u_{t} \in\left(\mathfrak{a}^{[p]}: \mathfrak{a}\right) \backslash \mathfrak{n}^{[p]}
$$

such that their natural images in $\left(\mathfrak{a}^{[p]}: \mathfrak{a}\right) / \mathfrak{a}^{[p]}$ form a generating set for this $S$ module; for each $i=1, \ldots, t$, let $E_{i}$ denote $\left(0:_{E} \mathfrak{a}\right)=E_{R}(R / \mathfrak{m})$ endowed with the (x-torsion-free) left $R[x, f]$-module structure for which $x e=u_{i}$ ye for all $e \in\left(0:_{E} \mathfrak{a}\right)$; and let $\mathfrak{b}_{i}$ be the unique smallest ideal of positive height in $\mathcal{I}\left(E_{i}\right)$. By [16, Theorem 3.5], each $\mathfrak{b}_{i}$ is contained in the test ideal $\tau(R)$ of $R$, so that $\mathfrak{b}:=\mathfrak{b}_{1}+\cdots+\mathfrak{b}_{t} \subseteq \tau(R)$. By Proposition [3.6. we have $\mathfrak{b} R_{c}=R_{c}$. Hence there exists $h \in \mathbb{N}$ such that $c^{h} \in \mathfrak{b} \subseteq \tau(R)$, and so there exists $e \in \mathbb{N}_{0}$ such that $c^{p^{e}}$ is a test element for $R$.

Therefore, for each finitely generated $R$-module $M$ and each $m \in 0_{M}^{*}$, we have $c^{p^{e}} x^{j}(1 \otimes m)=0$ in $R[x, f] \otimes_{R} M$, for all $j \in \mathbb{N}_{0}$. Thus, for $m \in 0_{M}^{*}$, we have

$$
x^{e} c x^{i}(1 \otimes m)=c^{p^{e}} x^{e+i}(1 \otimes m)=0 \quad \text { for all } i \in \mathbb{N}_{0} .
$$

However, the left $R[x, f]$-module $R[x, f] \otimes_{R} M$ is $x$-torsion-free. Hence $c x^{i}(1 \otimes m)=$ 0 for all $i \in \mathbb{N}_{0}$ and all $m \in 0_{M}^{*}$. As this is true for all finitely generated $R$-modules $M$, it follows that $c$ itself is a test element for $R$.

\section{Additional EmbedDing TheOrEms AND APPlicAtions TO $F$-PURE EXCELLENT RINGS}

In order to extend certain results from Section 3 to non-local $F$-pure rings, we are going to modify some constructions and arguments from [16. Suppose that $(R, \mathfrak{m})$ is local and $F$-pure, and endow $E:=E_{R}(R / \mathfrak{m})$ with a structure of an $x$-torsion-free left $R[x, f]$-module (Theorem 3.2 shows that this is possible). Let $M$ be a finitely generated $R$-module. In [16, Theorem 3.5], the author showed that the graded left $R[x, f]$-module $R[x, f] \otimes_{R} M$ can be embedded, by means of a homogeneous $R[x, f]$ monomorphism, into a product (in the category of $\mathbb{Z}$-graded left $R[x, f]$-modules and homogeneous homomorphisms) of countably many graded left $R[x, f]$-modules, each equal either to a certain graded left $R[x, f]$-module $\widetilde{E}$ constructed from $E$ (see [16. Lemma 2.4]) or to an extension (see [16, Definition 2.10]) of a shift of $\widetilde{E}$. In this paper, we are going to modify those ideas so that we can obtain a similar embedding for $R[x, f] \otimes_{R} L$ when $L$ is an arbitrary $R$-module. 
4.1. Definition. Let $H$ be a left $R[x, f]$-module, let $a \in R$ and let $\mathfrak{b}$ be an ideal of $R$. We say that $H$ is a-testable if, whenever $h \in H$ and $c \in R^{\circ}$ are such that $c x^{n} h=0$ for all $n \gg 0$, then $a x^{n} h=0$ for all $n \geq 0$.

It should be noted that there is no requirement that $a \in R^{\circ}$ in this definition.

Furthermore, we say that $H$ is $\mathfrak{b}$-testable if $H$ is $r$-testable for all $r \in \mathfrak{b}$. Suppose that $\mathfrak{b}$ can be generated by $b_{1}, \ldots, b_{w}$. Then it is clear that $H$ is $\mathfrak{b}$-testable if and only if it is $b_{i}$-testable for all $i=1, \ldots, w$.

Many of the uses of this concept will be in tight closure theory, in situations where the ideal $\mathfrak{b}$ has positive height. In this context, it should be noted that an ideal $\mathfrak{b}$ of positive height can be generated by (finitely many) elements of $\mathfrak{b} \cap R^{\circ}$; see [16, Lemma 3.4].

In this section, we shall make use of some facts about the behaviour under ring homomorphisms of certain concepts from [16].

4.2. Proposition. Let $\theta: R \longrightarrow R^{\prime}$ be a homomorphism of commutative Noetherian rings of characteristic $p$. Then $\theta$ induces a ring homomorphism $\widetilde{\theta}: R[x, f] \longrightarrow$ $R^{\prime}[x, f]$ for which $\widetilde{\theta}\left(\sum_{i=0}^{n} r_{i} x^{i}\right)=\sum_{i=0}^{n} \theta\left(r_{i}\right) x^{i}$ for all $n \in \mathbb{N}_{0}, r_{0}, \ldots, r_{n} \in R$.

Let $H^{\prime}$ be a left $R^{\prime}[x, f]$-module. Then $H^{\prime}$ can be regarded as a left $R[x, f]$-module by means of $\widetilde{\theta}$, and

(i) $\mathcal{A}_{R[x, f]}\left(H^{\prime}\right) \subseteq \mathcal{A}_{R^{\prime}[x, f]}\left(H^{\prime}\right)$;

(ii) $\mathcal{G}_{R[x, f]}\left(H^{\prime}\right)=\left\{\widetilde{\theta}^{-1}\left(\mathfrak{B}^{\prime}\right): \mathfrak{B}^{\prime} \in \mathcal{G}_{R^{\prime}[x, f]}\left(H^{\prime}\right)\right\}$;

(iii) in the special case in which $R^{\prime}=S^{-1} R$, where $S$ is a multiplicatively closed subset of $R$ and $\theta$ is the natural ring homomorphism $\xi: R \longrightarrow S^{-1} R$, then $\mathcal{A}_{R[x, f]}\left(H^{\prime}\right)=\mathcal{A}_{\left(S^{-1} R\right)[x, f]}\left(H^{\prime}\right)$.

Proof. Let ${ }^{e}$ stand for extension of ideals with respect to the ring homomorphism $\theta: R \longrightarrow R^{\prime}$.

(i), (iii) Let $G \in \mathcal{A}_{R[x, f]}\left(H^{\prime}\right)$. Thus there exists a graded two-sided ideal $\mathfrak{B}$ of $R[x, f]$ such that $G=\operatorname{ann}_{H^{\prime}}(\mathfrak{B})$. We can write $\mathfrak{B}=\bigoplus_{i \in \mathbb{N}_{0}} \mathfrak{b}_{i} x^{i}$ for a suitable ascending chain $\left(\mathfrak{b}_{i}\right)_{i \in \mathbb{N}_{0}}$ of ideals of $R$. Thus an element $h^{\prime} \in H^{\prime}$ belongs to $G$ if and only if, for all $i \in \mathbb{N}_{0}$ and all $r_{i} \in \mathfrak{b}_{i}$, we have $r_{i} x^{i} h^{\prime}=0$, that is, if and only if, for all $i \in \mathbb{N}_{0}$ and all $r_{i} \in \mathfrak{b}_{i}$, we have $\theta\left(r_{i}\right) x^{i} h^{\prime}=0$. Hence

$$
G=\operatorname{ann}_{H^{\prime}}\left(\bigoplus_{i \in \mathbb{N}_{0}}\left(\mathfrak{b}_{i}\right)^{e} x^{i}\right) \in \mathcal{A}_{R^{\prime}[x, f]}\left(H^{\prime}\right) .
$$

Now consider the special case in which $R^{\prime}=S^{-1} R$ and $\theta$ is the natural ring homomorphism $\xi$. Let $G^{\prime} \in \mathcal{A}_{\left(S^{-1} R\right)[x, f]}\left(H^{\prime}\right)$. Thus there exists a graded two-sided ideal $\mathfrak{B}^{\prime}$ of $\left(S^{-1} R\right)[x, f]$ such that $G^{\prime}=\operatorname{ann}_{H^{\prime}}\left(\mathfrak{B}^{\prime}\right)$. The fact that every ideal of $S^{-1} R$ is extended from its own contraction to $R$ means that there is an ascending chain $\left(\mathfrak{b}_{i}\right)_{i \in \mathbb{N}_{0}}$ of ideals of $R$ such that $\mathfrak{B}^{\prime}=\bigoplus_{i \in \mathbb{N}_{0}}\left(\mathfrak{b}_{i}\right)^{e} x^{i}$, and the argument in the immediately preceding paragraph shows that

$$
\operatorname{ann}_{H^{\prime}}\left(\bigoplus_{i \in \mathbb{N}_{0}} \mathfrak{b}_{i} x^{i}\right)=\operatorname{ann}_{H^{\prime}}\left(\bigoplus_{i \in \mathbb{N}_{0}}\left(\mathfrak{b}_{i}\right)^{e} x^{i}\right)=\operatorname{ann}_{H^{\prime}}\left(\mathfrak{B}^{\prime}\right)=G^{\prime}
$$

Therefore $G^{\prime} \in \mathcal{A}_{R[x, f]}\left(H^{\prime}\right)$.

(ii) Let $\mathfrak{B}^{\prime} \in \mathcal{G}_{R^{\prime}[x, f]}\left(H^{\prime}\right)$, so that $\mathfrak{B}^{\prime}=\operatorname{gr}^{\prime} \operatorname{ann}_{R^{\prime}[x, f]}\left(G^{\prime}\right)$ for some $R^{\prime}[x, f]$ submodule $G^{\prime}$ of $H^{\prime}$. Now $G^{\prime}$ is automatically an $R[x, f]$-submodule of $H^{\prime}$, and it is routine to check that $\operatorname{gr}-\operatorname{ann}_{R[x, f]}\left(G^{\prime}\right)=\widetilde{\theta}^{-1}\left(\mathfrak{B}^{\prime}\right)$. Thus we have proved that

$$
\mathcal{G}_{R[x, f]}\left(H^{\prime}\right) \supseteq\left\{\widetilde{\theta}^{-1}\left(\mathfrak{B}^{\prime}\right): \mathfrak{B}^{\prime} \in \mathcal{G}_{R^{\prime}[x, f]}\left(H^{\prime}\right)\right\} .
$$


To prove the reverse inclusion, let $\mathfrak{B} \in \mathcal{G}_{R[x, f]}\left(H^{\prime}\right)$. Then, by Lemma 1.2, we have $\mathfrak{B}=\operatorname{gr}^{-a n n} n_{R[x, f]} G$, where $G=\operatorname{ann}_{H^{\prime}}(\mathfrak{B}) \in \mathcal{A}_{R[x, f]}\left(H^{\prime}\right)$. By part (i), $G \in \mathcal{A}_{R^{\prime}[x, f]}\left(H^{\prime}\right)$, and so $G$ is an $R^{\prime}[x, f]$-submodule of $H^{\prime}$. Hence, by the first paragraph of this proof of part (ii),

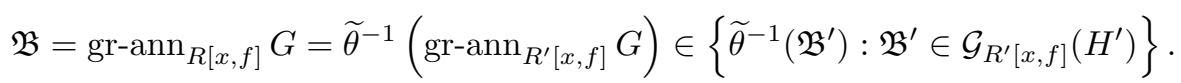

4.3. Notation. For the remainder of this section, $I$ will denote a non-empty, but possibly infinite, indexing set, we shall set $R_{i}:=R$ for all $i \in I$, and we shall use $V$ to denote the free $R$-module $\bigoplus_{i \in I} R_{i}$. We shall frequently use notation such as ' $\left(r_{i}\right)_{i \in I}$ ' to denote an element of $V$; when we do, it is of course to be understood that $r_{i} \neq 0$ for only finitely many $i \in I$.

We refer to the mapping $f: V \longrightarrow V$ for which $f\left(\left(r_{i}\right)_{i \in I}\right)=\left(r_{i}^{p}\right)_{i \in I}$ for all $\left(r_{i}\right)_{i \in I} \in V$ as the Frobenius map.

4.4. Lemma. Let $b \in \mathbb{N}$ and $W=\bigoplus_{n \geq b} W_{n}$ be a $\mathbb{Z}$-graded left $R[x, f]$-module; let $\left(g_{i}\right)_{i \in I}$ be a family of arbitrary elements of $W_{b}$ (so that infinitely many of them could be non-zero), indexed by the set I. Use the notation of \$4.3. Set

$$
K:=\left\{\left(r_{i}\right)_{i \in I} \in V: \sum_{i \in I} r_{i} g_{i}=0\right\},
$$

an $R$-submodule of $V$.

Then there is a graded left $R[x, f]$-module

$$
W^{\prime}=\bigoplus_{n \geq b-1} W_{n}^{\prime}=\left(V / f^{-1}(K)\right) \oplus W_{b} \oplus W_{b+1} \oplus \cdots \oplus W_{t} \oplus \cdots
$$

(so that $W_{b-1}^{\prime}=V / f^{-1}(K)$ and $W_{n}^{\prime}=W_{n}$ for all $n \geq b$ ) which has $W$ as an $R[x, f]$ submodule and for which $x\left(\left(r_{i}\right)_{i \in I}+f^{-1}(K)\right)=\sum_{i \in I} r_{i}^{p} g_{i}$ for all $\left(r_{i}\right)_{i \in I} \in V$. We call $W^{\prime}$ the 1 -place extension of $W$ by $\left(g_{i}\right)_{i \in I}$, and denote it by $\operatorname{exten}\left(W ;\left(g_{i}\right)_{i \in I} ; 1\right)$.

Let $a \in R$. If $W$ is a-testable, then so too is $\operatorname{exten}\left(W ;\left(g_{i}\right)_{i \in I} ; 1\right)$.

If $W$ is $x$-torsion-free, then so too is $\operatorname{exten}\left(W ;\left(g_{i}\right)_{i \in I} ; 1\right)$, and then

$$
\mathcal{G}\left(\operatorname{exten}\left(W ;\left(g_{i}\right)_{i \in I} ; 1\right)\right)=\mathcal{G}(W)
$$

and $\mathcal{I}\left(\operatorname{exten}\left(W ;\left(g_{i}\right)_{i \in I} ; 1\right)\right)=\mathcal{I}(W)$.

Proof. All the claims in this lemma, except the one that $W^{\prime}:=\operatorname{exten}\left(W ;\left(g_{i}\right)_{i \in I} ; 1\right)$ is $a$-testable if $W$ is, can be proved in a manner entirely similar to that used to prove [16, Lemma 2.8].

So suppose that $W$ is $a$-testable. To show that $W^{\prime}$ is $a$-testable, it is enough for us to show that, whenever $w$ is a homogeneous element of $W^{\prime}$ and $c \in R^{\circ}$ are such that $c x^{n} w=0$ for all $n \gg 0$, then $w$ is annihilated by $R a R[x, f]$. This is immediate from the fact that $W$ is $a$-testable when $w$ has degree at least $b$. We therefore suppose that $w \in W_{b-1}^{\prime}$, say $w=\left(r_{i}\right)_{i \in I}+f^{-1}(K)$ for some $\left(r_{i}\right)_{i \in I} \in V$. Since $c x^{n}(x w)=0$ for all $n \gg 0$ and $x w \in W_{b}$, it follows that $x w$ is annihilated by $\operatorname{RaR}[x, f]$; therefore $a x^{n} w=0$ for all $n \geq 1$. It remains to show that $w$ is annihilated by $a$.

Now $x a w=a^{p} x w=0$. Therefore

$$
0=x a w=x\left(\left(a r_{i}\right)_{i \in I}+f^{-1}(K)\right)=\sum_{i \in I} a^{p} r_{i}^{p} g_{i},
$$


so that $\left(a r_{i}\right)_{i \in I} \in f^{-1}(K)$ and $a w=0$. Therefore $w$ is annihilated by $a$, and so by $\operatorname{RaR}[x, f]$.

4.5. Definition. Let $b \in \mathbb{N}$ and $W=\bigoplus_{n \geq b} W_{n}$ be a $\mathbb{Z}$-graded left $R[x, f]$-module, and let $\left(g_{i}\right)_{i \in I}$ be a family of arbitrary elements of $W_{b}$, as in Lemma 4.4. The 1-place extension exten $\left(W ;\left(g_{i}\right)_{i \in I} ; 1\right)$ of $W$ by $\left(g_{i}\right)_{i \in I}$ was defined in Lemma 4.4 Recall that, with the notation of 4.3 we defined

$$
K:=\left\{\left(r_{i}\right)_{i \in I} \in V: \sum_{i \in I} r_{i} g_{i}=0\right\} .
$$

Now let $h \in \mathbb{N}$ with $h \geq 2$. The $h$-place extension $\operatorname{exten}\left(W ;\left(g_{i}\right)_{i \in I} ; h\right)$ of $W$ by $\left(g_{i}\right)_{i \in I}$ is the graded left $R[x, f]$-module

$$
\left(V / f^{-h}(K)\right) \oplus \cdots \oplus\left(V / f^{-1}(K)\right) \oplus W_{b} \oplus \cdots \oplus W_{t} \oplus \cdots
$$

which has exten $\left(W ;\left(g_{i}\right)_{i \in I} ; 1\right)$ as a graded $R[x, f]$-submodule and is such that

$$
x\left(v+f^{-j}(K)\right)=f(v)+f^{-(j-1)}(K) \text { for all } v \in V \text { and } j=h, h-1, \ldots, 2 .
$$

For each $j \in I$, let $e_{j}$ denote the element $\left(r_{i}\right)_{i \in I}$ of $V$ for which $r_{j}=1$ and $r_{i}=0$ for all $i \in I \backslash\{j\}$. It is straightforward to check that

$$
\operatorname{exten}\left(W ;\left(g_{i}\right)_{i \in I} ; h\right)=\operatorname{exten}\left(\operatorname{exten}\left(W ;\left(g_{i}\right)_{i \in I} ; 1\right) ;\left(\overline{e_{i}}\right)_{i \in I} ; h-1\right),
$$

where, for $v \in V$, we use $\bar{v}$ to denote $v+f^{-1}(K)$, and

$$
\operatorname{exten}\left(W ;\left(g_{i}\right)_{i \in I} ; h\right)=\operatorname{exten}\left(\operatorname{exten}\left(W ;\left(g_{i}\right)_{i \in I} ; h-1\right) ;\left(\widetilde{e_{i}}\right)_{i \in I} ; 1\right),
$$

where, for $v \in V$, we use $\widetilde{v}$ to denote $v+f^{-(h-1)}(K)$.

Let $a \in R$. It is a consequence of Lemma 4.4 that, if $W$ is $a$-testable, then so too is $\operatorname{exten}\left(W ;\left(g_{i}\right)_{i \in I} ; h\right)$.

It is also a consequence of Lemma 4.4 that, if $W$ is $x$-torsion-free, then so too is $\operatorname{exten}\left(W ;\left(g_{i}\right)_{i \in I} ; h\right)$, and then $\mathcal{G}\left(\operatorname{exten}\left(W ;\left(g_{i}\right)_{i \in I} ; h\right)\right)=\mathcal{G}(W)$ and

$$
\mathcal{I}\left(\operatorname{exten}\left(W ;\left(g_{i}\right)_{i \in I} ; h\right)\right)=\mathcal{I}(W) .
$$

Finally, it will occasionally be convenient to extend the terminology and regard $W$ itself as a 0-place extension of $W$.

4.6. Proposition. Let $b \in \mathbb{N}$ and $W=\bigoplus_{n>b} W_{n}$ be a $\mathbb{Z}$-graded left $R[x, f]$-module. Use the notation of 4.3. Let $\left\{m_{i}: i \in \bar{I}\right\}$ be a generating set for an $R$-module $M$. We can form the graded $R[x, f]$-submodule $\bigoplus_{n \geq b}\left(R x^{n} \otimes_{R} M\right)$ of $R[x, f] \otimes_{R} M$. Suppose that there is given a homogeneous $R[x, f]$-homomorphism $\lambda^{\prime}=\bigoplus_{n \geq b} \lambda_{n}$ : $\bigoplus_{n \geq b}\left(R x^{n} \otimes_{R} M\right) \longrightarrow W$.

For each $i \in I$, let $g_{i}:=\lambda_{b}\left(x^{b} \otimes m_{i}\right) \in W_{b}$. Set

$$
K:=\left\{\left(r_{i}\right)_{i \in I} \in V: \sum_{i \in I} r_{i} g_{i}=0\right\}
$$

as in Lemma 4.4. For each $n=0,1, \ldots, b-1$, there exists an $R$-homomorphism $\lambda_{n}: R x^{n} \otimes_{R} M \longrightarrow V / f^{-(b-n)}(K)$ such that

$$
\lambda_{n}\left(\sum_{i \in I} r_{i} x^{n} \otimes m_{i}\right)=\left(r_{i}\right)_{i \in I}+f^{-(b-n)}(K) \quad \text { for all }\left(r_{i}\right)_{i \in I} \in V .
$$

Furthermore,

$$
\lambda:=\bigoplus_{n \in \mathbb{N}_{0}} \lambda_{n}: R[x, f] \otimes_{R} M=\bigoplus_{n \in \mathbb{N}_{0}}\left(R x^{n} \otimes_{R} M\right) \longrightarrow \operatorname{exten}\left(W ;\left(g_{i}\right)_{i \in I} ; b\right)
$$

is a homogeneous $R[x, f]$-homomorphism that extends $\lambda^{\prime}$. 
Proof. Let $n \in\{0, \ldots, b-1\}$. Note that the $R$-module $R x^{n} \otimes_{R} M$ is generated by $\left\{x^{n} \otimes m_{i}: i \in I\right\}$. Now if $\left(r_{i}\right)_{i \in I},\left(s_{i}\right)_{i \in I} \in V$ are such that $\sum_{i \in I} r_{i} x^{n} \otimes m_{i}=$ $\sum_{i \in I} s_{i} x^{n} \otimes m_{i}$, then multiplication of both sides on the left by $x^{b-n}$ yields that $\sum_{i \in I} r_{i}^{p^{b-n}} x^{b} \otimes m_{i}=\sum_{i \in I} s_{i}^{p^{b-n}} x^{b} \otimes m_{i}$. Apply the $R$-homomorphism $\lambda_{b}$ to deduce that

$$
\begin{aligned}
\sum_{i \in I}\left(r_{i}-s_{i}\right)^{p^{b-n}} g_{i} & =\sum_{i \in I}\left(r_{i}^{p^{b-n}}-s_{i}^{p^{b-n}}\right) \lambda_{b}\left(x^{b} \otimes m_{i}\right) \\
& =\lambda_{b}\left(\sum_{i \in I} r_{i}^{p^{b-n}} x^{b} \otimes m_{i}-\sum_{i \in I} s_{i}^{p^{b-n}} x^{b} \otimes m_{i}\right)=\lambda_{b}(0)=0 .
\end{aligned}
$$

Therefore $\left(r_{i}-s_{i}\right)_{i \in I} \in f^{-(b-n)}(K)$, and

$$
\left(r_{i}\right)_{i \in I}+f^{-(b-n)}(K)=\left(s_{i}\right)_{i \in I}+f^{-(b-n)}(K) .
$$

After this, all the remaining claims are easy to check.

4.7. Reminders. We shall need to use two constructions from [16, §2], and we include reminders here for the reader's convenience.

(i) Let $\left(H^{(\lambda)}\right)_{\lambda \in \Lambda}$ be a non-empty family of $\mathbb{Z}$-graded left $R[x, f]$-modules, with gradings given by $H^{(\lambda)}=\bigoplus_{n \in \mathbb{Z}} H_{n}^{(\lambda)}$ for each $\lambda \in \Lambda$. The $R$-module

$$
\prod_{\lambda \in \Lambda}^{\prime} H^{(\lambda)}:=\bigoplus_{n \in \mathbb{Z}}\left(\prod_{\lambda \in \Lambda} H_{n}^{(\lambda)}\right)
$$

is a (Z्Z-graded) left $R[x, f]$-module in which

$$
x\left(h_{n}^{(\lambda)}\right)_{\lambda \in \Lambda}=\left(x h_{n}^{(\lambda)}\right)_{\lambda \in \Lambda} \in \prod_{\lambda \in \Lambda} H_{n+1}^{(\lambda)} \quad \text { for all }\left(h_{n}^{(\lambda)}\right)_{\lambda \in \Lambda} \in \prod_{\lambda \in \Lambda} H_{n}^{(\lambda)} .
$$

In this paper, we shall refer to $\prod_{\lambda \in \Lambda}^{\prime} H^{(\lambda)}$ as the graded product of the $H^{(\lambda)}$.

Let $a \in R$. It is clear that if $H^{(\lambda)}$ is $a$-testable for all $\lambda \in \Lambda$, then $\prod_{\lambda \in \Lambda}^{\prime} H^{(\lambda)}$ is also $a$-testable.

(ii) Let $H$ be a left $R[x, f]$-module. For all $n \in \mathbb{N}_{0}$, set $H_{n}:=H$. Then the $R$-module $\widetilde{H}:=\bigoplus_{n \in \mathbb{N}_{0}} H_{n}$ has a natural structure as a graded left $R[x, f]$-module under which the result of multiplying $h_{n} \in H_{n}=H$ on the left by $x$ is the element $x h_{n} \in H_{n+1}=H$. In this paper, we shall refer to $\widetilde{H}$ as the graded companion of $H$.

Let $a \in R$. It is clear that if $H$ is $a$-testable, then so too is $\widetilde{H}$.

(iii) Let $\left(L^{(\lambda)}\right)_{\lambda \in \Lambda}$ be a non-empty family of (ungraded) left $R[x, f]$-modules. Then it is easy to check that

$$
\prod_{\lambda \in \Lambda}^{\prime} \widetilde{L^{(\lambda)}}=\widetilde{\prod_{\lambda \in \Lambda} L^{(\lambda)}}
$$

Thus, speaking loosely, the operations of taking products and graded companions commute.

4.8. Lemma. Let $G:=\bigoplus_{n \in \mathbb{N}_{0}} G_{n}$ be a positively graded $x$-torsion-free left $R[x, f]$ module for which $\mathcal{I}(G)$ is a finite set, and let $\mathfrak{b}$ be the smallest ideal of positive height 
in $\mathcal{I}(G)$. Then any graded product $K$ of extensions of shifts of graded products of copies of $G$ is again $x$-torsion-free, has $\mathcal{I}(K)=\mathcal{I}(G)$, and is $\mathfrak{b}$-testable.

For example, if $E$ is an $x$-torsion-free left $R[x, f]$-module for which $\mathcal{I}(E)$ is a finite set, and $\mathfrak{d}$ denotes the smallest ideal of positive height in $\mathcal{I}(E)$, then any graded product $L$ of extensions of shifts of graded products of copies of the graded companion $\widetilde{E}$ of $E$ is again $x$-torsion-free, has $\mathcal{I}(L)=\mathcal{I}(E)$, and is $\mathfrak{d}$-testable.

Proof. It follows from Lemma 4.4 that the process of extension preserves torsionfreeness and the set of special $R$-ideals; it is clear that shifting also preserves torsion-freeness and the set of special $R$-ideals; and [16, Lemma 2.3] shows that the graded product, say $K$, of a non-empty family of $x$-torsion-free $\mathbb{Z}$-graded left $R[x, f]$-modules which all have the same set $\mathcal{I}$ of special $R$-ideals is again $x$-torsionfree with $\mathcal{I}(K)=\mathcal{I}$. If we denote by $\mathfrak{b}$ the smallest ideal of positive height in $\mathcal{I}$, then it follows from [15, Theorem 3.12] that $K$ is $\mathfrak{b}$-testable.

Finally, the process of passing from $E$ to its graded companion $\widetilde{E}$ preserves torsion-freeness and the set of special $R$-ideals, by [16, Lemma 2.5], and so the claims in the final paragraph of the lemma follow on application of the first paragraph to $\widetilde{E}$.

The next lemma is a generalization of Lemma 3.1 of [16].

4.9. Lemma. Suppose that $(R, \mathfrak{m})$ is local and that there exists an $\mathbb{N}_{0}$-graded left $R[x, f]$-module $G=\bigoplus_{n \in \mathbb{N}_{0}} G_{n}$ such that $G_{0}$ is $R$-isomorphic to $E_{R}(R / \mathfrak{m})$, the injective envelope of the simple $R$-module $R / \mathfrak{m}$.

Let $M$ be an $\mathfrak{m}$-torsion $R$-module. Then the injective envelope $E_{R}(M)$ of $M$ is $R$-isomorphic to a direct sum of copies of $E_{R}(R / \mathfrak{m})$, say to $\bigoplus_{j \in J} E^{(j)}$, where $E^{(j)}=G_{0}$ for all $j$ in an indexing set $J$. Set $G^{(j)}=G$ for all $j \in J$. Then there exists a homogeneous $R[x, f]$-homomorphism

$$
\lambda:=\bigoplus_{n \in \mathbb{N}_{0}} \lambda_{n}: R[x, f] \otimes_{R} M=\bigoplus_{n \in \mathbb{N}_{0}}\left(R x^{n} \otimes_{R} M\right) \longrightarrow \prod_{j \in J}^{\prime} G^{(j)},
$$

such that $\lambda_{0}$ is a monomorphism.

Proof. The fact that $E_{R}(M)$ is $R$-isomorphic to a direct sum of copies of $E_{R}(R / \mathfrak{m})$ follows from E. Matlis's theory of injective modules over a commutative Noetherian ring, described in (for example) [14, Chapter 18]. Set

$$
L=\bigoplus_{n \in \mathbb{N}_{0}} L_{n}:=\prod_{j \in J}^{\prime} G^{(j)} .
$$

Since $\bigoplus_{j \in J} G_{0}^{(j)}$ can be embedded in $\prod_{j \in J} G_{0}^{(j)}$, there is an $R$-monomorphism $\lambda_{0}: M \longrightarrow L_{0}$. We can define, for each $n \in \mathbb{N}_{0}$, an $R$-homomorphism

$$
\lambda_{n}: R x^{n} \otimes_{R} M \longrightarrow L_{n}
$$

such that $\lambda_{n}\left(r x^{n} \otimes m\right)=r x^{n} \lambda_{0}(m)$ for all $r \in R$ and all $m \in M$. It is straightforward to check that the $\lambda_{n}\left(n \in \mathbb{N}_{0}\right)$ provide a homogeneous $R[x, f]$-homomorphism as claimed.

We are now able to prove an embedding theorem, for $\mathfrak{m}$-torsion modules over the local ring $(R, \mathfrak{m})$, that is reminiscent of the result in the first part of [16, Theorem $3.5]$. 
4.10. Theorem. Suppose that $(R, \mathfrak{m})$ is local, and that there exists an $\mathbb{N}_{0}$-graded left $R[x, f]$-module $G=\bigoplus_{n \in \mathbb{N}_{0}} G_{n}$ such that $G_{0}$ is $R$-isomorphic to $E_{R}(R / \mathfrak{m})$, the injective envelope of the simple $R$-module $R / \mathfrak{m}$. Let $M$ be an $\mathfrak{m}$-torsion $R$-module. Then there is a family $\left(H^{(n)}\right)_{n \in \mathbb{N}_{0}}$ of $\mathbb{N}_{0}$-graded left $R[x, f]$-modules, where $H^{(n)}$ is an $n$-place extension of a shift of a graded product of copies of $G$ (for each $n \in \mathbb{N}_{0}$ ), for which there exists a homogeneous $R[x, f]$-monomorphism

$$
\nu: R[x, f] \otimes_{R} M=\bigoplus_{i \in \mathbb{N}_{0}}\left(R x^{i} \otimes_{R} M\right) \longrightarrow \prod_{n \in \mathbb{N}_{0}}^{\prime} H^{(n)}=: K .
$$

If $G$ is $x$-torsion-free and $\mathcal{I}(G)$ is finite, and if we let $\mathfrak{b}$ be the smallest ideal of positive height in $\mathcal{I}(G)$, then $K$ is x-torsion-free with $\mathcal{I}(K)=\mathcal{I}(G)$ and $R[x, f] \otimes_{R} M$ is $\mathfrak{b}$-testable.

Proof. For each $n \in \mathbb{N}_{0}$, the (left) $R$-module $R x^{n} \otimes_{R} M$ is $\mathfrak{m}$-torsion, since, if $\mathfrak{m}^{t} g=0$ for a $g \in M$ and some $t \in \mathbb{N}$, then $\left(\mathfrak{m}^{t}\right)^{\left[p^{n}\right]}\left(x^{n} \otimes g\right)=0$.

Let $n \in \mathbb{N}_{0}$. By Lemma 4.9, there is a family $\left(G^{(j, n)}\right)_{j \in Y_{n}}$ of graded left $R[x, f]$ modules, all equal to $G$, and a homogeneous $R[x, f]$-homomorphism

$$
R[x, f] \otimes_{R}\left(R x^{n} \otimes_{R} M\right) \longrightarrow \prod_{j \in Y_{n}}^{\prime} G^{(j, n)}
$$

which is monomorphic in degree 0 . If we now use isomorphisms of the type described in [16, Remark 3.2], we obtain (after application of the shift functor $(\bullet)(-n)$ ) a homogeneous $R[x, f]$-homomorphism

$$
\zeta^{(n)}: \bigoplus_{j \geq n}\left(R x^{j} \otimes_{R} M\right) \longrightarrow\left(\prod_{j \in Y_{n}}{ }^{\prime(j, n)}\right)(-n)
$$

which is monomorphic in degree $n$. We can now use Proposition 4.6 to extend $\zeta^{(n)}$ by $n$ places to produce a homogeneous $R[x, f]$-homomorphism

$$
\lambda^{(n)}: \bigoplus_{j \geq 0}\left(R x^{j} \otimes_{R} M\right)=R[x, f] \otimes_{R} M \longrightarrow H^{(n)},
$$

where $H^{(n)}$ is an appropriate $n$-place extension of $\left(\prod_{j \in Y_{n}}^{\prime} G^{(j, n)}\right)(-n)$, such that $\lambda^{(n)}$ is monomorphic in degree $n$.

There is therefore a homogeneous $R[x, f]$-homomorphism

$$
\nu=\bigoplus_{j \in \mathbb{N}_{0}} \nu_{j}: R[x, f] \otimes_{R} M \longrightarrow \prod_{n \in \mathbb{N}_{0}}^{\prime} H^{(n)}=: K
$$

such that $\nu_{j}\left(\xi_{j}\right)=\left(\left(\lambda^{(n)}\right)_{j}\left(\xi_{j}\right)\right)_{n \in \mathbb{N}_{0}}$ for all $j \in \mathbb{N}_{0}$ and $\xi_{j} \in R x^{j} \otimes_{R} M$. For each $j \in \mathbb{N}_{0}$, we have that $\left(\lambda^{(j)}\right)_{j}$ is a monomorphism; hence $\nu_{j}$ is a monomorphism. Hence $\nu$ is an $R[x, f]$-monomorphism.

Lemma 4.8 shows that $K$ is $x$-torsion-free with $\mathcal{I}(K)=\mathcal{I}(G)$, and that $K$ is $\mathfrak{b}$-testable. In view of the $R[x, f]$-monomorphism $\nu$, it follows that $R[x, f] \otimes_{R} M$ is $\mathfrak{b}$-testable.

4.11. Corollary. Suppose that $(R, \mathfrak{m})$ is F-pure and local. Then the left $R[x, f]$ module $R[x, f] \otimes_{R} E_{R}(R / \mathfrak{m})$ is $x$-torsion-free, and its set $\mathcal{I}\left(R[x, f] \otimes_{R} E_{R}(R / \mathfrak{m})\right)$ of $\left(R[x, f] \otimes_{R} E_{R}(R / \mathfrak{m})\right)$-special $R$-ideals is finite. 
In fact, for any $x$-torsion-free left $R[x, f]$-module structure on $E_{R}(R / \mathfrak{m})$ that extends its $R$-module structure (and such exist, by Theorem 3.2), we have

$$
\mathcal{I}\left(R[x, f] \otimes_{R} E_{R}(R / \mathfrak{m})\right) \subseteq \mathcal{I}\left(E_{R}(R / \mathfrak{m})\right),
$$

and the latter set is finite; furthermore, if $\mathfrak{b}$ is the smallest ideal in $\mathcal{I}\left(E_{R}(R / \mathfrak{m})\right)$ of positive height, then $R[x, f] \otimes_{R} E_{R}(R / \mathfrak{m})$ is $\mathfrak{b}$-testable.

Proof. In view of Theorem 3.2, it is enough to prove the claim in the second paragraph. So select an $x$-torsion-free left $R[x, f]$-module structure on $E:=E_{R}(R / \mathfrak{m})$ that extends its $R$-module structure. Construct the graded companion $\widetilde{E}$ of $E$, as in Reminders 4.7 (ii), and recall that $\widetilde{E}$ is again $x$-torsion-free and has $\mathcal{I}(\widetilde{E})=\mathcal{I}(E)$. Since $E$ is $\mathfrak{m}$-torsion, it follows from Theorem 4.10 that there exists an $x$-torsion-free $\mathbb{N}_{0}$-graded left $R[x, f]$-module $K$ with $\mathcal{I}(K)=\mathcal{I}(E)$, and a homogeneous $R[x, f]$ monomorphism $\nu: R[x, f] \otimes_{R} E=\bigoplus_{i \in \mathbb{N}_{0}}\left(R x^{i} \otimes_{R} E\right) \longrightarrow K$. Therefore $R[x, f] \otimes_{R} E$ is $x$-torsion-free and $\mathcal{I}\left(R[x, f] \otimes_{R} E\right) \subseteq \mathcal{I}(E)$. It follows from [15, Corollary 3.11] that $\mathcal{I}(E)$ is finite; we can then use [15. Theorem 3.12] to deduce that $K$ and $R[x, f] \otimes_{R} E$ are $\mathfrak{b}$-testable.

4.12. Corollary. Suppose that $(R, \mathfrak{m})$ is F-pure, local and complete. Let $c \in R^{\circ}$ be such that $R_{c}$ is regular. Let $M$ be an $\mathfrak{m}$-torsion $R$-module. Then the left $R[x, f]$ module $R[x, f] \otimes_{R} M$ is $x$-torsion-free and c-testable.

Proof. We appeal to Cohen's Structure Theorem for complete local rings containing a subfield and write $R=S / \mathfrak{a}$, where $S$ is a complete regular local ring of characteristic $p$ and $\mathfrak{a}$ is a proper, non-zero ideal of $S$. This is consistent with the notation of 42.1 , and we shall use that notation for the remainder of this proof.

We now appeal to Proposition 3.6. Use Lemma 3.5 to find

$$
u_{1}, \ldots, u_{t} \in\left(\mathfrak{a}^{[p]}: \mathfrak{a}\right) \backslash \mathfrak{n}^{[p]}
$$

such that their natural images in $\left(\mathfrak{a}^{[p]}: \mathfrak{a}\right) / \mathfrak{a}^{[p]}$ form a generating set for this $S$ module; for each $i=1, \ldots, t$, let $E_{i}$ denote $\left(0:_{E} \mathfrak{a}\right)=E_{R}(R / \mathfrak{m})$ endowed with the (x-torsion-free) left $R[x, f]$-module structure for which $x e=u_{i} y e$ for all $e \in\left(0:_{E} \mathfrak{a}\right)$; and let $\mathfrak{b}_{i}$ be the unique smallest ideal of positive height in $\mathcal{I}\left(E_{i}\right)$.

By Theorem 4.10 (applied to the graded companions $\widetilde{E_{1}}, \ldots, \widetilde{E_{t}}$ in turn), we deduce that $R[x, f] \otimes_{R} M$ is $x$-torsion-free, and $\mathfrak{b}_{i}$-testable for all $i=1, \ldots, t$. Therefore $R[x, f] \otimes_{R} M$ is $\left(\mathfrak{b}_{1}+\cdots+\mathfrak{b}_{t}\right)$-testable. By Proposition 3.6, we have $\left(\mathfrak{b}_{1}+\cdots+\mathfrak{b}_{t}\right) R_{c}=R_{c}$. Hence some power of $c$ belongs to $\mathfrak{b}_{1}+\cdots+\mathfrak{b}_{t}$, and there exists $e \in \mathbb{N}_{0}$ such that $R[x, f] \otimes_{R} M$ is $c^{p^{e}}$-testable.

Therefore, for each $y \in R[x, f] \otimes_{R} M$ for which there exists $d \in R^{\circ}$ such that $d x^{n} y=0$ for all $n \gg 0$, we have $c^{p^{e}} x^{j} y=0$ for all $j \in \mathbb{N}_{0}$. Thus

$$
x^{e} c x^{i} y=c^{p^{e}} x^{e+i} y=0 \text { for all } i \in \mathbb{N}_{0} .
$$

However, $R[x, f] \otimes_{R} M$ is $x$-torsion-free, and so $c x^{i} y=0$ for all $i \in \mathbb{N}_{0}$. Therefore $R[x, f] \otimes_{R} M$ is $c$-testable.

4.13. Corollary. Suppose that $(R, \mathfrak{m})$ is F-pure, local and excellent. Let $c \in R^{\circ}$ be such that $R_{c}$ is regular. Let $M$ be an $\mathfrak{m}$-torsion $R$-module. Then the left $R[x, f]$ module $R[x, f] \otimes_{R} M$ is $x$-torsion-free and c-testable.

In particular, $R[x, f] \otimes_{R} E_{R}(R / \mathfrak{m})$ is $x$-torsion-free and c-testable. 
Proof. Note that $\widehat{R}$ is again F-pure, by Lemma 1.7. Complete local rings are always excellent. Furthermore, the fibre rings of the flat ring homomorphism $R_{c} \longrightarrow \widehat{R}_{c}$ induced by inclusion are rings of fractions of the formal fibres of $R$, and so are regular; it therefore follows that $\widehat{R}_{c}$ is regular. Note that $c \in \widehat{R}^{\circ}$.

We now appeal to Corollary 4.12 to deduce that the graded left $\widehat{R}[x, f]$-module

$$
\widehat{R}[x, f] \otimes_{\widehat{R}} E_{\widehat{R}}(\widehat{R} / \mathfrak{m} \widehat{R})
$$

is $x$-torsion-free and $c$-testable. Regard this as an $R[x, f]$-module in the natural way: it is still $x$-torsion-free and $c$-testable, and its 0 th component is $R$-isomorphic to $E_{R}(R / \mathfrak{m})$.

By Theorem 4.10, there is a family $\left(H^{(n)}\right)_{n \in \mathbb{N}_{0}}$ of $\mathbb{N}_{0}$-graded left $R[x, f]$-modules, where $H^{(n)}$ is an $n$-place extension of a shift of a graded product of copies of

$$
\widehat{R}[x, f] \otimes_{\widehat{R}} E_{\widehat{R}}(\widehat{R} / \mathfrak{m} \widehat{R})
$$

(for each $n \in \mathbb{N}_{0}$ ), for which there exists a homogeneous $R[x, f]$-monomorphism

$$
\nu: R[x, f] \otimes_{R} M=\bigoplus_{i \in \mathbb{N}_{0}}\left(R x^{i} \otimes_{R} M\right) \longrightarrow \prod_{n \in \mathbb{N}_{0}}^{\prime} H^{(n)}=: K .
$$

By Lemma 4.4 and Reminders $4.7(\mathrm{i})$, the left $R[x, f]$-module $K$ is $x$-torsion-free and $c$-testable, and therefore so too is $R[x, f] \otimes_{R} M$.

The final claim now follows from the fact that $E_{R}(R / \mathfrak{m})$ is an $\mathfrak{m}$-torsion $R$ module.

4.14. Corollary. Suppose that $R$ is F-pure and excellent (but not necessarily local). Let $c \in R^{\circ}$ be such that $R_{c}$ is regular.

Let $\mathfrak{p} \in \operatorname{Spec}(R)$, and let $k(\mathfrak{p})$ denote the simple $R_{\mathfrak{p}}$-module. View the left $R_{\mathfrak{p}}[x, f]$-module

$$
R_{\mathfrak{p}}[x, f] \otimes_{R_{\mathfrak{p}}} E_{R_{\mathfrak{p}}}(k(\mathfrak{p}))
$$

as an $R[x, f]$-module in the natural way. Then $R_{\mathfrak{p}}[x, f] \otimes_{R_{\mathfrak{p}}} E_{R_{\mathfrak{p}}}(k(\mathfrak{p}))$ is $x$-torsionfree and c-testable.

Proof. By Lemma 1.7 the localization $R_{\mathfrak{p}}$ is again $F$-pure; of course, $R_{\mathfrak{p}}$ is excellent. Furthermore, since $\left(R_{\mathfrak{p}}\right)_{c / 1}$, the ring of fractions of $R_{\mathfrak{p}}$ with respect to the set of powers of the element $c / 1$ of $\left(R_{\mathfrak{p}}\right)^{\circ}$, is a ring of fractions of $R_{c}$, it is regular.

We can now appeal to Corollary 4.13 to deduce that the left $R_{\mathfrak{p}}[x, f]$-module $R_{\mathfrak{p}}[x, f] \otimes_{R_{\mathfrak{p}}} E_{R_{\mathfrak{p}}}(k(\mathfrak{p}))$ is $x$-torsion-free and $(c / 1)$-testable. Therefore, when we regard $R_{\mathfrak{p}}[x, f] \otimes_{R_{\mathfrak{p}}} E_{R_{\mathfrak{p}}}(k(\mathfrak{p}))$ as an $R[x, f]$-module in the natural way, it is $x$ torsion-free and $c$-testable.

4.15. Lemma. For each $\mathfrak{p} \in \operatorname{Spec}(R)$, let $k(\mathfrak{p})$ denote the simple $R_{\mathfrak{p}}$-module and let

$$
H(\mathfrak{p}):=R_{\mathfrak{p}}[x, f] \otimes_{R_{\mathfrak{p}}} E_{R_{\mathfrak{p}}}(k(\mathfrak{p})),
$$

viewed as a graded left $R[x, f]$-module in the natural way. Observe that the 0th component of $H(\mathfrak{p})$ is $R$-isomorphic to $E_{R}(R / \mathfrak{p})$.

Let $M$ be an $R$-module, and express its injective envelope $E_{R}(M)$ as a direct sum of indecomposable injective $R$-modules, say $E_{R}(M) \cong \bigoplus_{j \in J} E_{R}\left(R / \mathfrak{p}^{(j)}\right)$, where $\left(\mathfrak{p}^{(j)}\right)_{j \in J}$ is an appropriate family of prime ideals of $R$. (The superscript ${ }^{(j)}$ here is 
an index and does not indicate symbolic power.) Then there exists a homogeneous $R[x, f]$-homomorphism

$$
\lambda:=\bigoplus_{n \in \mathbb{N}_{0}} \lambda_{n}: R[x, f] \otimes_{R} M=\bigoplus_{n \in \mathbb{N}_{0}}\left(R x^{n} \otimes_{R} M\right) \longrightarrow \prod_{j \in J}^{\prime} H\left(\mathfrak{p}^{(j)}\right),
$$

such that $\lambda_{0}$ is a monomorphism.

Proof. Set

$$
L=\bigoplus_{n \in \mathbb{N}_{0}} L_{n}:=\prod_{j \in J}^{\prime} H\left(\mathfrak{p}^{(j)}\right) .
$$

Since $\bigoplus_{j \in J} H\left(\mathfrak{p}^{(j)}\right)_{0} \cong \bigoplus_{j \in J} E_{R}\left(R / \mathfrak{p}^{(j)}\right)$ and $\bigoplus_{j \in J} H\left(\mathfrak{p}^{(j)}\right)_{0}$ can be embedded in $\prod_{j \in J} H\left(\mathfrak{p}^{(j)}\right)_{0}$, there is an $R$-monomorphism $\lambda_{0}: M \longrightarrow L_{0}$. We can define, for each $n \in \mathbb{N}_{0}$, an $R$-homomorphism $\lambda_{n}: R x^{n} \otimes_{R} M \longrightarrow L_{n}$ such that $\lambda_{n}\left(r x^{n} \otimes m\right)=$ $r x^{n} \lambda_{0}(m)$ for all $r \in R$ and all $m \in M$. It is straightforward to check that the $\lambda_{n}\left(n \in \mathbb{N}_{0}\right)$ provide a homogeneous $R[x, f]$-homomorphism as claimed.

We are now in a position to prove the main result of this paper.

4.16. Theorem. For each $\mathfrak{p} \in \operatorname{Spec}(R)$, let $k(\mathfrak{p})$ denote the simple $R_{\mathfrak{p}}$-module and let

$$
H(\mathfrak{p}):=R_{\mathfrak{p}}[x, f] \otimes_{R_{\mathfrak{p}}} E_{R_{\mathfrak{p}}}(k(\mathfrak{p})),
$$

viewed as a graded left $R[x, f]$-module in the natural way.

Let $M$ be an R-module. Then there is a family $\left(G^{(n)}\right)_{n \in \mathbb{N}_{0}}$ of $\mathbb{N}_{0}$-graded left $R[x, f]$-modules, where $G^{(n)}$ is an n-place extension of a shift of a graded product of graded left $R[x, f]$-modules of the form $H(\mathfrak{p})$ for various prime ideals $\mathfrak{p}$ of $R$ (for each $n \in \mathbb{N}_{0}$ ), for which there exists a homogeneous $R[x, f]$-monomorphism

$$
\nu: R[x, f] \otimes_{R} M=\bigoplus_{i \in \mathbb{N}_{0}}\left(R x^{i} \otimes_{R} M\right) \longrightarrow \prod_{n \in \mathbb{N}_{0}}^{\prime} G^{(n)} .
$$

If $R$ is excellent and $F$-pure, and $c \in R^{\circ}$ is such that $R_{c}$ is regular, then $R[x, f] \otimes_{R} M$ is c-testable; as this is true for each $R$-module $M$, it follows that $c$ is a big test element for $R$.

Thus if $R$ is excellent and F-pure, then $R$ has a big test element.

Proof. Let $n \in \mathbb{N}_{0}$. By Lemma 4.15, there is a family $\left(\mathfrak{p}^{(j, n)}\right)_{j \in Y_{n}}$ of prime ideals of $R$, and a homogeneous $R[x, f]$-homomorphism

$$
R[x, f] \otimes_{R}\left(R x^{n} \otimes_{R} M\right) \longrightarrow \prod_{j \in Y_{n}}^{\prime} H\left(\mathfrak{p}^{(j, n)}\right)
$$

which is monomorphic in degree 0 . If we now use isomorphisms of the type described in [16, Remark 3.2], we obtain (after an application of the shift functor $(\bullet)(-n)$ ) a homogeneous $R[x, f]$-homomorphism

$$
\zeta^{(n)}: \bigoplus_{j \geq n}\left(R x^{j} \otimes_{R} M\right) \longrightarrow\left(\prod_{j \in Y_{n}}^{\prime} H\left(\mathfrak{p}^{(j, n)}\right)\right)(-n)
$$

which is monomorphic in degree $n$. We can now use Proposition 4.6 to extend $\zeta^{(n)}$ by $n$ places to produce a homogeneous $R[x, f]$-homomorphism

$$
\lambda^{(n)}: \bigoplus_{j \geq 0}\left(R x^{j} \otimes_{R} M\right)=R[x, f] \otimes_{R} M \longrightarrow G^{(n)},
$$


where $G^{(n)}$ is an appropriate $n$-place extension of $\left(\prod_{j \in Y_{n}}^{\prime} H\left(\mathfrak{p}^{(j, n)}\right)\right)(-n)$, such that $\lambda^{(n)}$ is monomorphic in degree $n$.

There is therefore a homogeneous $R[x, f]$-homomorphism

$$
\nu=\bigoplus_{j \in \mathbb{N}_{0}} \nu_{j}: R[x, f] \otimes_{R} M=\bigoplus_{j \in \mathbb{N}_{0}}\left(R x^{j} \otimes_{R} M\right) \longrightarrow \prod_{n \in \mathbb{N}_{0}}^{\prime} G^{(n)}=: K
$$

such that $\nu_{j}\left(\xi_{j}\right)=\left(\left(\lambda^{(n)}\right)_{j}\left(\xi_{j}\right)\right)_{n \in \mathbb{N}_{0}}$ for all $j \in \mathbb{N}_{0}$ and $\xi_{j} \in R x^{j} \otimes_{R} M$. For each $j \in \mathbb{N}_{0}$, we have that $\left(\lambda^{(j)}\right)_{j}$ is a monomorphism; hence $\nu_{j}$ is a monomorphism. Hence $\nu$ is an $R[x, f]$-monomorphism.

Now suppose that $R$ is excellent and $F$-pure, and that $c \in R^{\circ}$ is such that $R_{c}$ is regular. By Corollary 4.14, the left $R[x, f]$-module $H(\mathfrak{p})$ is $x$-torsion-free and $c$ testable. It now follows from Lemma 4.4 and Reminders 4.7 that $\prod_{j \in Y_{n}}^{\prime} H\left(\mathfrak{p}^{(j, n)}\right)$ is $x$-torsion-free and $c$-testable for all $n \in \mathbb{N}_{0}$, that $G^{(n)}$ is $x$-torsion-free and $c$-testable for all $n \in \mathbb{N}_{0}$, and that $K$ is $x$-torsion-free and $c$-testable.

Therefore $R[x, f] \otimes_{R} M$ is ( $x$-torsion-free and) $c$-testable for each $R$-module $M$, and so $c$ is a big test element for $R$.

Experts in tight closure theory will know that it is desirable to have 'locally stable' or 'completely stable' test elements. These are defined as follows.

4.17. Definitions. A test element for modules (respectively, for ideals) $c$ for $R$ is said to be a locally stable test element for modules (respectively, for ideals) for $R$ if and only if, for every $\mathfrak{p} \in \operatorname{Spec}(R)$, the natural image $c / 1$ of $c$ in $R_{\mathfrak{p}}$ is a test element for modules (respectively, for ideals) for $R_{\mathfrak{p}}$.

A test element for modules (respectively, for ideals) $c$ for $R$ is said to be a completely stable test element for modules (respectively, for ideals) for $R$ if and only if, for every $\mathfrak{p} \in \operatorname{Spec}(R)$, the natural image $c / 1$ of $c$ in $\widehat{R_{\mathfrak{p}}}$ is a test element for modules (respectively, for ideals) for $\widehat{R_{\mathfrak{p}}}$.

We make corresponding definitions for big test elements.

4.18. Definitions. A big test element $c$ for $R$ is said to be a locally stable (respectively, completely stable) big test element for $R$ if and only if, for every $\mathfrak{p} \in \operatorname{Spec}(R)$, the natural image $c / 1$ of $c$ in $R_{\mathfrak{p}}$ (respectively, $\widehat{R_{\mathfrak{p}}}$ ) is a big test element for $R_{\mathfrak{p}}$ (respectively, for $\left.\widehat{R_{\mathfrak{p}}}\right)$.

We can now use Theorem 4.16 to obtain big test elements that are locally stable and completely stable.

4.19. Corollary. Let $R$ be excellent and F-pure, and let $c \in R^{\circ}$ be such that $R_{c}$ is regular. Then $c$ is a locally stable big test element for $R$ and a completely stable big test element for $R$.

Proof. We proved in Theorem 4.16 that $c$ is a big test element for $R$. Let $\mathfrak{p} \in$ $\operatorname{Spec}(R)$. Of course, $c / 1 \in R_{\mathfrak{p}}$ belongs to both $\left(R_{\mathfrak{p}}\right)^{\circ}$ and $\left(\widehat{R_{\mathfrak{p}}}\right)^{\circ}$.

By Lemma 1.7, the localization $R_{\mathfrak{p}}$ and its completion $\widehat{R_{\mathfrak{p}}}$ are again $F$-pure; of course, $R_{\mathfrak{p}}$ and $\widehat{R_{\mathfrak{p}}}$ are excellent. Furthermore, since $\left(R_{\mathfrak{p}}\right)_{c / 1}$, the ring of fractions of $R_{\mathfrak{p}}$ with respect to the set of powers of the element $c / 1$ of $\left(R_{\mathfrak{p}}\right)^{\circ}$, is a ring of fractions of $R_{c}$, it is regular. In addition, the fibre rings of the flat ring homomorphism 
$\left(R_{\mathfrak{p}}\right)_{c / 1} \longrightarrow\left(\widehat{R_{\mathfrak{p}}}\right)_{c / 1}$ induced by inclusion are rings of fractions of the formal fibres of $R_{\mathfrak{p}}$, and so are regular; it therefore follows that $\left(\widehat{R_{\mathfrak{p}}}\right)_{c / 1}$ is regular.

We can therefore use Theorem 4.16 to deduce that $c / 1$ is a big test element for both $R_{\mathfrak{p}}$ and $\widehat{R_{\mathfrak{p}}}$.

\section{REFERENCES}

[1] M. Blickle, The intersection homology D-module in finite characteristic, Ph.D. dissertation, University of Michigan, Ann Arbor, 2001.

[2] M. P. Brodmann and R. Y. Sharp, Local cohomology: An algebraic introduction with geometric applications, Cambridge Studies in Advanced Mathematics 60, Cambridge University Press, 1998. MR 1613627 (99h:13020)

[3] R. Fedder, F-purity and rational singularity, Transactions Amer. Math. Soc. 278 (1983) 461-480. MR701505 (84h:13031)

[4] R. Hartshorne and R. Speiser, Local cohomological dimension in characteristic p, Annals of Math. 105 (1977) 45-79. MR0441962 (56:353)

[5] M. Hochster and C. Huneke, Tight closure, invariant theory and the Briançon-Skoda Theorem, J. Amer. Math. Soc. 3 (1990) 31-116. MR1017784 (91g:13010)

[6] M. Hochster and C. Huneke, F-regularity, test elements, and smooth base change, Transactions Amer. Math. Soc. 346 (1994) 1-62. MR1273534 (95d:13007)

[7] M. Hochster and J. L. Roberts, Rings of invariants of reductive groups acting on regular rings are Cohen-Macaulay, Advances in Math. 13 (1974) 115-175. MR0347810 (50:311)

[8] C. Huneke, Tight closure and its applications, Conference Board of the Mathematical Sciences Regional Conference Series in Mathematics 88, American Mathematical Society, Providence, 1996. MR.1377268 (96m:13001)

[9] C. Huneke and R. Y. Sharp, Bass numbers of local cohomology modules, Transactions Amer. Math. Soc. 339 (1993) 765-779. MR 1124167 (93m:13008)

[10] M. Katzman, Parameter-test-ideals of Cohen-Macaulay rings, Compositio Math. 144 (2008) 933-948. MR2441251 (2009d:13030)

[11] M. Katzman and R. Y. Sharp, Uniform behaviour of the Frobenius closures of ideals generated by regular sequences, J. Algebra 295 (2006) 231-246. MR2188859(2006i:13007)

[12] G. Lyubeznik, F-modules: Applications to local cohomology and D-modules in characteristic $p>0$, J. reine angew. Math. 491 (1997) 65-130. MR.1476089 (99c:13005)

[13] G. Lyubeznik and K. E. Smith, On the commutation of the test ideal with localization and completion, Transactions Amer. Math. Soc. 353 (2001) 3149-3180. MR/1828602 (2002f:13010)

[14] H. Matsumura, Commutative ring theory, Cambridge Studies in Advanced Mathematics 8, Cambridge University Press, 1986. MR 879273 (88h:13001)

[15] R. Y. Sharp, Graded annihilators of modules over the Frobenius skew polynomial ring, and tight closure, Transactions Amer. Math. Soc. 359 (2007) 4237-4258. MR2309183 (2008b:13006)

[16] R. Y. Sharp, Graded annihilators and tight closure test ideals, J. Algebra 322 (2009) 34103426. MR2567428

[17] R. Y. Sharp and N. Nossem, Ideals in a perfect closure, linear growth of primary decompositions, and tight closure, Transactions Amer. Math. Soc. 356 (2004) 3687-3720. MR 2055750 (2005a:13009)

[18] D. W. Sharpe and P. Vámos, Injective modules, Cambridge Tracts in Mathematics and Mathematical Physics 62, Cambridge University Press, 1972. MR0360706 (50:13153)

[19] K. E. Smith, F-rational rings have rational singularities, Amer. J. Math. 119 (1997) 159-180. MR1428062 (97k:13004)

Department of Pure Mathematics, University of Sheffield, Hicks Building, Sheffield, S3 7RH, UNITED KINGDOM

E-mail address: R.Y.Sharp@sheffield.ac.uk 